\title{
Wilson loops on Riemann surfaces, Liouville theory and covariantization of the conformal group
}

\author{
Marco Matone and Paolo Pasti \\ Dipartimento di Fisica e Astronomia "G. Galilei" and INFN, \\ Università di Padova, Via Marzolo, 8-35131 Padova, Italy \\ E-mail: matone@pd.infn.it, pasti@pd.infn.it
}

ABSTRACT: The covariantization procedure is usually referred to the translation operator, that is the derivative. Here we introduce a general method to covariantize arbitrary differential operators, such as the ones defining the fundamental group of a given manifold. We focus on the differential operators representing the $\mathrm{sl}_{2}(\mathbb{R})$ generators, which in turn, generate, by exponentiation, the two-dimensional conformal transformations. A key point of our construction is the recent result on the closed forms of the Baker-Campbell-Hausdorff formula. In particular, our covariantization receipt is quite general. This has a deep consequence since it means that the covariantization of the conformal group is always definite. Our covariantization receipt is quite general and apply in general situations, including AdS/CFT. Here we focus on the projective unitary representations of the fundamental group of a Riemann surface, which may include elliptic points and punctures, introduced in the framework of noncommutative Riemann surfaces. It turns out that the covariantized conformal operators are built in terms of Wilson loops around Poincaré geodesics, implying a deep relationship between gauge theories on Riemann surfaces and Liouville theory.

KEYwords: Gauge Symmetry, Conformal Field Models in String Theory, AdS-CFT Correspondence

ARXiv EPrint: 1505.02569 


\section{Contents}

1 Introduction 1

2 Differential representation of the conformal group 5

2.1 Uniformization and Liouville equation 5

$\begin{array}{lll}2.2 & \text { Differential representation of } \mathrm{sl}_{2}(\mathbb{R}) & 7\end{array}$

2.3 Parameters of the representation 8

3 Parameterization of $\mathrm{SL}_{2}(\mathbb{R})$ by the closed forms of the $\mathrm{BCH}$ formula 10

4 Setting the problem $\quad 12$

4.1 A first screening 13

4.2 An alternative to the $\mathrm{BCH}$ formula on the torus 14

$\begin{array}{lll}4.3 & \text { The hidden invariance } & 16\end{array}$

5 Unitary projective representations of the Fuchsian group 18

$\begin{array}{ll}5.1 \text { The problem of simultaneous covariantization } & 18\end{array}$

$\begin{array}{lll}5.2 & \text { The unitary covariantized operators } & 21\end{array}$

5.3 Selecting the Fuchsian generators 23

$\begin{array}{lll}5.4 & \text { The Wilson-Fuchs operators } & 25\end{array}$

5.5 Covariantizing the generators of the uniformizing group 27

5.6 Computing the phase 28

$\begin{array}{lll}5.7 & \text { Nonabelian extension } & 30\end{array}$

6 The gauge length 31

$\begin{array}{lll}6.1 & \text { The gauge length as pseudo-distance } & 32\end{array}$

6.2 The gauge length as Poincaré area 32

6.3 Möbius transformations as gauge transformations 34

\section{Introduction}

It is well-known that in $2 D$ space-time a pure gauge theory is locally trivial. In particular, by $\partial_{\mu} F^{\mu \nu}=0$, it follows that the unique nontrivial component $E_{1}=F_{01}$ is a constant and the finite energy condition fixes $E_{1}=0$. This is a consequence of the fact that the number of degrees of freedom of a pure gauge theory in $D$-dimensions is $D-2$. Essentially, in topologically trivial 2D space-time any gauge configuration can be absorbed by a gauge transformation.

The situation is completely different in the case one considers non-trivial topologies. In particular, there are well-known models corresponding to a topological field theory with a 
strict relationship with string theory. Consider the case of a $\mathrm{SU}(N)$ (or $\mathrm{U}(N)$ ) Yang-Mills theory on a genus $g$ Riemann surface $\Sigma$

$$
\mathcal{Z}_{\Sigma}=\int D A^{\mu} \exp \left[-\frac{1}{4 g^{2}} \int_{\Sigma} d^{2} x \sqrt{\operatorname{det} g} \operatorname{tr} F_{\mu \nu} F^{\mu \nu}\right]
$$

where the trace is on the fundamental representation. Cutting $\Sigma$ along a basis of its fundamental group $\pi_{1}(\Sigma)$, one gets the relation

$$
a_{1} b_{1} a_{1}^{-1} b_{1}^{-1} \ldots a_{g} b_{g} a_{g}^{-1} b_{g}^{-1}=I
$$

It turns out that the partition function can be expressed in terms of path integral of the traces of matrices of the gauge group associated to each generator of $\pi_{1}(\Sigma)$ and (see [1] and references therein)

$$
\mathcal{Z}_{\Sigma}=\sum_{R} d_{R} e^{-\frac{g A c_{2}(R)}{2}} \int \prod D U_{l} D V_{l} \operatorname{tr}_{R}\left[U_{1} V_{1} U_{1}^{+} V_{1}^{+} \ldots U_{g} V_{g} U_{g}^{+} V_{g}^{+}\right]
$$

where the summation is over the irreducible representations of the group, $A$ is the area of $\Sigma, d_{R}$ the dimension of the representation $R$ and $c_{2}(R)$ the second Casimir operator of $R$.

The relation between connections and the fundamental group of $\Sigma$ appears also in the context of noncommutative Riemann surfaces. This corresponds to consider unitary projective representations of the uniformizing Fuchsian groups. Such representations are obtained by covariantizing the differential operators of the $\mathrm{sl}_{2}(\mathbb{R})$ algebra. Roughly speaking, whereas the projective unitary representation of the (abelian) group uniformizing the torus

$$
\mathcal{U}_{1} \mathcal{U}_{2}=e^{2 \pi i \theta} \mathcal{U}_{2} \mathcal{U}_{1}
$$

is simply obtained by setting $\mathcal{U}_{k}=\exp \left(\lambda_{k}\left(\partial_{k}+i A_{k}\right)\right)$, with $A$ a connection 1-form, in the case of uniformizing groups one needs to covariantize the generators of $\operatorname{sl}_{2}(\mathbb{R})$. In other words, first one has to find the receipe to covariantize $\partial_{z}, z \partial_{z}$ and $z^{2} \partial_{z}$ and then finding the unitary operators $\mathcal{U}_{k}$ such that

$$
\prod_{k=1}^{g} \mathcal{U}_{2 k-1} \mathcal{U}_{2 k} \mathcal{U}_{2 k-1}^{-1} \mathcal{U}_{2 k}^{-1}=e^{2 \pi i \theta} I
$$

This case corresponds to the one of hyperbolic Riemann surfaces. However, we will consider the general case that includes Riemann surfaces with elliptic point and punctures.

We will see that finding the unitary projective representation connects several questions, such as the one of the simultaneous covariantization, originally considered in our previous work $[2,3]$, that now are investigated in a systematic way and solved step by step. In our investigation, we will express the Möbius transformations in terms of the differential representation of $\operatorname{sl}_{2}(\mathbb{R})$. This is done by first expressing and element of $\mathrm{PSL}_{2}(\mathbb{R})$ as the composition of a translation, dilatation and a special conformal transformation. However, this has a drawback for our purposes. The reason is that the order of the above composition may change according to the kind of Möbius transformation one is considering. This is 
related to the important question of expressing $\mathcal{U}_{k}$ as the exponential of a unique operator, conjugated by a functional $F_{k}$ of the connection $A$, associated to the geodesic defining the corresponding generators of the uniformizing group, namely

$$
\mathcal{U}_{k}=F_{k}(z, \bar{z}) \exp \left(D_{k}\right) F_{k}^{-1}(z, \bar{z}) .
$$

The problem then is to find $D_{k}$ and the functional $F_{k}$, which is the key object to covariantize $D_{k}$. As we will see, such questions are in turn related to the problem of finding the explicit relation between the normal form of $\pi_{1}(\Sigma)$ and the uniformizing group $\Gamma$ of $\Sigma$. We will find such a relation, that selects the corresponding form of the generators $\beta_{k}$ of $\Gamma$. As such, these generators satisfy the relation

$$
\beta_{4 g} \beta_{4 g-1} \ldots \beta_{1}=I,
$$

which is the case of compact Riemann surfaces of genus $g$ (our investigation extends to the case with elliptic points and punctures). As we will see, all the above questions, unanswered in $[2,3]$, have a solution which is essentially unique.

The operator $\exp \left(D_{k}\right)$ performs the Möbius transformation $\beta_{k}^{-1}$ of the arguments of a function, so that

$$
\mathcal{U}_{k}=F_{k}(z, \bar{z}) F_{k}^{-1}\left(\beta_{k}^{-1} z, \beta_{k}^{-1} \bar{z}\right) \exp \left(D_{k}\right)
$$

where

$$
\beta_{k} z:=\frac{a_{k} z+b_{k}}{c_{k} z+d_{k}} .
$$

The $F_{k}$ 's are directly related to the Wilson loop associated to the geodesic connecting $z$ and $\beta_{k}^{-1} z$ on the upper half plane $\mathbb{H}$, namely

$$
F_{k}(z, \bar{z}) F_{k}^{-1}\left(\beta_{k}^{-1} z, \beta_{k}^{-1} \bar{z}\right)=\exp \left(i b \int_{z}^{\beta_{k}^{-1} z} A\right)
$$

where $b$ is a real parameter. Therefore,

$$
\mathcal{U}_{k}=\exp \left(i b \int_{z}^{\beta_{k}^{-1} z} A\right) \exp \left(D_{k}\right) .
$$

Note that

$$
W_{\beta_{k}}=\exp \left(i b \int_{z}^{\beta_{k}^{-1} z} A\right),
$$

projects to a Wilson loop on $\Sigma$. We call the $\mathcal{U}_{k}$ 's, Wilson-Fuchs operators. The modulus of

$$
d_{A}(z, w)=\int_{z}^{w} A
$$

related to the Wilson loop by $W_{\beta_{k}}=\exp \left(i b d_{A}\left(z, \beta_{k}^{-1}\right)\right)$, is a pseudo distance that called "gauge-length" in $[2,3]$. It corresponds to the Poincaré area of the hyperbolic triangle whose sides are the geodesic joining $z$ and $w$, together with the two geodesics connecting $z$ and $w$ to the point at imaginary infinity on the upper half-plane. An outcome of $[2,3]$ is that Möbius transformations correspond to gauge transformations. 
As we said, the above construction is possible once one finds $D_{k}$ such that any Möbius transformation can expressed in the form $\exp \left(D_{k}\right)$. Such a question is equivalent to the problem of finding the closed form of $W$ such that

$$
\exp (X) \exp (Y) \exp (Z)=\exp (W)
$$

The recent solution of such a problem is a key point of our construction. In particular, it turns out that our covariantization receipt is quite general. This has a deep consequence since it means that the covariantization of the conformal group is always definite. In [4], it has been introduced an algorithm to derive the closed form of $W$ in (1.11) for a wide class of commutator algebras, classified in [5] and applied to all semisimple complex Lie algebras in [6]. The algorithm in [4], that extends the remarkable result by Van-Brunt and Visser [7] (see also [8] for related results), exploits the associative property of the Baker-CampbellHausdorff formula and implementing in it the Jacobi identity. In particular, it turns out that when $X, Y$ and $Z$ are elements of $\operatorname{sl}_{2}(\mathbb{R})$, the corresponding commutator algebras is, according to the classification in [5], a subtype of the type 4. As a result, we will see that the Möbius transformation (1.6) is represented by the unitary operator $\mathcal{U}_{k}=\exp \left(\mathcal{D}_{k}\right)$, where $\mathcal{D}_{k}$ is the $\operatorname{sl}_{2}(\mathbb{R})$ covariantized operator

$$
\begin{aligned}
& \mathcal{D}_{k}=\frac{\lambda_{+}^{(k)}-\lambda_{-}^{(k)}}{e^{-\lambda_{-}^{(k)}}-e^{-\lambda_{+}^{(k)}}} \times \\
& \times F_{k}\left[\lambda_{-1}^{(k)}\left(\partial_{z}+\partial_{\bar{z}}\right)+\left(2-e^{-\lambda_{+}^{(k)}}-e^{-\lambda_{-}^{(k)}}\right)\left(z \partial_{z}+\bar{z} \partial_{\bar{z}}+1\right)\right. \\
& \left.+\lambda_{1}^{(k)}\left(z^{2} \partial_{z}+\bar{z}^{2} \partial_{\bar{z}}+z+\bar{z}\right)\right] F_{k}^{-1},
\end{aligned}
$$

where the parameters $\lambda_{ \pm}^{(k)}$ and $\lambda_{j}^{(k)}, j=-1,01$, are defined in terms of the components of the matrix $\beta_{k}$.

Usually, the covariantization procedure is considered only for the translation operator, that is for the ordinary derivative. The above covariantization is the one for the conformal transformations. To derive (1.12) it has been introduced a general method to covariantize much more general operators than $\partial_{z}$. Actually, our analysis starts with the torus, whose fundamental domain are straight lines, and these are generated by derivatives. The noncommutative torus leads to a covariantization of the derivatives that apparently cannot be extended to the higher genus case. However, we reformulate the noncommutative torus in a more geometrical way. It turns out that such a geometrical formulation is the natural framework to derive, in analogy with the case of the torus, the corresponding quantities for negatively curved Riemann surfaces. Such a strategy provides the correct prescription to define the covariantized operators and leads to (1.12).

The above covariantization prescription can be extended to more general cases, including AdS/CFT. In particular whenever a manifold is naturally associated to a differential representation of its fundamental group. Here we focus on the projective unitary representations of the fundamental group of a Riemann surface, which may include elliptic points and punctures, introduced in the framework of noncommutative Riemann surfaces. 
The organization of the paper is as follows. In section 2, after shortly reviewing the uniformization theorem, we express the Möbius transformations in terms of the exponentiation of differential operators representing $\mathrm{sl}_{2}(\mathbb{R})$. In section 3 we express the action of such operators in the form of the exponential of a linear combination of the $\mathrm{sl}_{2}(\mathbb{R})$ generators. This is a basic step to formulate the covariantization of the conformal group. In section 4 we first reformulate the noncommutative torus in a more geometrical form, and find a hidden symmetry. Next, in section 5 , such a geometrical analysis will be used to formulate the unitary projective representation of the group uniformizing arbitrary Riemann surfaces. In particular, we will focus on the problem of simultaneous covariantization of the differential operators representing the $\mathrm{sl}_{2}(\mathbb{R})$ generatoras and of their complex conjugate. This will lead to the covariantization of the conformal operators as given in (1.12). We will also solve the problem of finding the explicit relation between the normal form of $\pi_{1}(\Sigma)$ and the uniformizing group $\Gamma$ of $\Sigma$. The extension to the nonabelian case is introduced in subsection 5.7. Section 6 is devoted to the properties of the gauge length as pseudo-distance and will show that it corresponds to a Poincaré area.

\section{Differential representation of the conformal group}

In this section, after shortly reviewing the uniformization theorem for Riemann surfaces, we express the $\mathrm{PSL}_{2}(\mathbb{R})$ transformations, acting on the upper half-plane, in terms of the composition of three exponentiations of the generators of $\operatorname{sl}_{2}(\mathbb{R})$, represented by the differential operators $\ell_{k}=z^{k+1} \partial_{z}, k=-1,0,1$. This will lead to consider two questions. The first is that such a prescription is not general, since the order of the composition depends on the specific Möbius transformation. The other question is related to the problem of expressing the unitary operators $\mathcal{U}_{k}$ in the form of a unique exponential

$$
\mathcal{U}_{k}=\exp \left(\mathcal{D}_{k}\right)
$$

As we will see, this is the natural way to define the covariantization of the conformal group. Such preliminary questions are solved in the next section, where we will derive the form of a Möbius transformation in the form of a unique exponential, whose argument is a linear combination of the $\ell_{k}$ 's.

\subsection{Uniformization and Liouville equation}

In the following, given a $2 \times 2$ matrix

$$
\mu=\left(\begin{array}{ll}
a & b \\
c & d
\end{array}\right),
$$

we adopt the notation

$$
\mu z=\frac{a z+b}{c z+d} .
$$

Let $\mathbb{D}$ be either the Riemann sphere $\hat{\mathbb{C}}=\mathbb{C} \cup\{\infty\}$, the complex plane $\mathbb{C}$, or the upper half-plane

$$
\mathbb{H}=\{z \in \mathbb{C} \mid \Im(z)>0\}
$$


According to the uniformization theorem, every Riemann surface $\Sigma$ is conformally equivalent to $\mathbb{D} / \Gamma$, where $\Gamma$ is a freely acting discontinuous group of fractional transformations preserving $\mathbb{D}$. Let $J_{\mathbb{H}}$ be the uniformizing map $J_{\mathbb{H}}: \mathbb{H} \longrightarrow \Sigma$. It has the invariance property

$$
J_{\mathbb{H}}(\gamma z)=J_{\mathbb{H}}(z),
$$

$\gamma \in \Gamma$, where $\Gamma \subset \mathrm{PSL}_{2}(\mathbb{R})=\mathrm{SL}_{2}(\mathbb{R}) /\{ \pm I\}$ is a finitely generated Fuchsian group. It acts on $\mathbb{H}$ by the linear fractional transformations

$$
\gamma z=\frac{a z+b}{c z+d} \in \mathbb{H}, \quad \gamma=\left(\begin{array}{ll}
a & b \\
c & d
\end{array}\right) \in \Gamma,
$$

$z \in \mathbb{H}$. By the fixed point equation $\gamma z=z$, that is

$$
z_{ \pm}=\frac{a-d \pm \sqrt{(a+d)^{2}-4}}{2 c}
$$

it follows that there are three kinds of $\mathrm{PSL}_{2}(\mathbb{R})$ matrices.

1. Elliptic: $|\operatorname{tr} \gamma|<2$. Then $\gamma$ has one fixed point on $\mathbb{H}\left(z_{-}=\bar{z}_{+} \notin \mathbb{R}\right)$ and $\Sigma$ has a branched point $w_{-}=J_{\mathbb{H}}\left(z_{-}\right)$. The finite order of its stabilizer defines its index $n \in \mathbb{N} \backslash\{0,1\}$. If $\Gamma$ contains elliptic elements, then $\mathbb{H} / \Gamma$ is an orbifold.

2. Parabolic: $|\operatorname{tr} \gamma|=2$. Then $z_{-}=z_{+} \in \mathbb{R}$ and the point $J_{\mathbb{H}}\left(z_{-}\right)$corresponds to a missing point of $\Sigma$, i.e. a puncture. The order of the stabilizer is now infinite.

3. Hyperbolic: $|\operatorname{tr} \gamma|>2$. The fixed points are distinct and lie on $\mathbb{R}=\partial \mathbb{H}$. Such $\gamma$ 's correspond to handles of $\Sigma$ and can be expressed in the form ${ }^{1}$

$$
\frac{\gamma z-z_{+}}{\gamma z-z_{-}}=e^{\lambda} \frac{z-z_{+}}{z-z_{-}}
$$

$e^{\lambda} \in \mathbb{R} \backslash\{0,1\}$.

The Poincaré metric on $\mathbb{H}$ is the metric with scalar curvature -1

$$
d s^{2}=\frac{|d z|^{2}}{(\Im(z))^{2}} .
$$

This implies that the Liouville equation on $\Sigma$

$$
\partial_{\bar{w}} \partial_{w} \varphi=\frac{e^{\varphi}}{2},
$$

has the unique solution

$$
e^{\varphi}=\frac{\left|J_{\mathbb{H}}^{-1}(w)^{\prime}\right|^{2}}{\left(\Im J_{\mathbb{H}}^{-1}(w)\right)^{2}} .
$$

\footnotetext{
${ }^{1}$ Given $z$ and $\gamma z, \lambda(\gamma)$ corresponds to the minimal distance between them. This minimum is reached for $z$ lying on the geodesic intersecting the real axis at $z_{-}$and $z_{+}$.
} 
The basic property of the Poincaré metric is that its isometry group $\mathrm{PSL}_{2}(\mathbb{R})$ coincides with the automorphism group of $\mathbb{H}$. The group $\Gamma$ is isomorphic to the fundamental group $\pi_{1}(\Sigma)$.

If $\Gamma$ uniformizes a surface of genus $g$ with $n$ punctures and $m$ elliptic points with indices $2 \leq n_{1} \leq n_{2} \leq \ldots \leq n_{m}<\infty$, then $\Gamma$ is generated by $2 g$ hyperbolic elements $\gamma_{1}, \ldots, \gamma_{2 g}, m$ elliptic elements $E_{1}, \ldots, E_{m}$, and $n$ parabolic elements $P_{1}, \ldots, P_{n}$ satisfying the relations

$$
E_{j}^{n_{j}}=I, \quad\left(\prod_{l=1}^{m} E_{l}\right)\left(\prod_{k=1}^{n} P_{k}\right) \prod_{j=1}^{g}\left(\gamma_{2 j-1} \gamma_{2 j} \gamma_{2 j-1}^{-1} \gamma_{2 j}^{-1}\right)=I .
$$

The uniformizing group carries information both on the topological and the complex structures of the Riemann surface. It is easy to see that the number of parameters fixing the generators of a Fuchsian group coincides with the dimension of moduli space of complex structures of Riemann surfaces. For example, in the case of compact Riemann surfaces of genus $g$, the full set of generators depends on $6 g$ real parameters which reduce to $6 g-3$ upon using (2.5). On the other hand, (2.5) remains invariant under conjugation by an element of $\mathrm{SL}_{2}(\mathbb{R})$ leading to $3 g-3$ complex parameters.

\subsection{Differential representation of $\operatorname{sl}_{2}(\mathbb{R})$}

Let $\ell_{n}, n=-1,0,1$, be the generators of $\operatorname{sl}_{2}(\mathbb{R})$. Fix their normalization by

$$
\left[\ell_{m}, \ell_{n}\right]=(n-m) \ell_{m+n},
$$

$n=-1,0,1$. Consider the representation $\ell_{n} \mapsto z^{n+1} \partial_{z} \in \operatorname{End}\left(\mathbb{C}\left[z, z^{-1}\right]\right)$. Thus, we set

$$
\ell_{n}=z^{n+1} \partial_{z}
$$

Note that $\left[\ell_{n}, f\right]=z^{n+1} \partial_{z} f$. Each element of $\mathrm{PSL}_{2}(\mathbb{R})$ can be expressed as the composition of a translation, dilatation and a special conformal transformation. These $\mathrm{PSL}_{2}(\mathbb{R})$ transformations are

$$
\exp \left(\lambda_{-1} \ell_{-1}\right) z=z+\lambda_{-1}, \quad \exp \left(\lambda_{0} \ell_{0}\right) z=e^{\lambda_{0}} z, \quad \exp \left(\lambda_{1} \ell_{1}\right) z=\frac{z}{1-\lambda_{1} z} .
$$

If $f(z)$ admits a convergent series expansion, then

$$
\exp \left(\lambda_{j} \ell_{j}\right) f(z)=f\left(\exp \left(\lambda_{j} \ell_{j}\right) z\right)
$$

We note that this action can be defined by considering the formal Taylor theorem. This corresponds to equating the application of a formal exponential of a formal multiple of $\partial_{z}$ with a formal substitution operation. For example,

$$
\exp \left(\lambda_{-1} \ell_{-1}\right) f(z)=f\left(z+\lambda_{-1}\right) .
$$

In this case $f(z)$ is an arbitrary series, including the formal ones. In particular, this series may have the form $\sum_{n} a_{n} z^{n}$, with $n$ which may also take complex values. Note that the formal expansion of $\exp \left(\lambda_{-1} \ell_{-1}\right)$, should not be confused with the standard meaning of formal series expansion, referring to non-convergent expansions. In particular, note that 
given an element $X$ of a Lie algebra $\mathfrak{g}$, equipped with a norm $\|\cdot\|, \exp (X)$ is well defined on all $\mathbb{C}$ whenever $\|X\|$ is finite.

Recall that

$$
\mu_{j}\left(\mu_{k} z\right)=\left(\mu_{j} \mu_{k}\right)(z)
$$

and set $h_{j}(z)=\exp \left(\lambda_{j} \ell_{j}\right) z$. Since

$$
\exp \left(\lambda_{k} \ell_{k}\right) \exp \left(\lambda_{j} \ell_{j}\right) f(z)=\exp \left(\lambda_{k} \ell_{k}\right) f\left(h_{j}(z)\right)=f\left(h_{j}\left(h_{k}(z)\right)\right)
$$

it follows that $\exp \left(\lambda_{k} \ell_{k}\right) \exp \left(\lambda_{j} \ell_{j}\right)$ acts in reverse order with respect to the matrix representation. This implies that the representation $V$ constructed in terms of the above differential operators acts as have

$$
V(\mu) f(z)=f\left(\mu^{-1} z\right)
$$

so that, since

$$
V(\mu) V(\nu) f(z)=f\left(\nu^{-1} \mu^{-1} z\right)=f\left((\mu \nu)^{-1} z\right)=V(\mu \nu) f(z),
$$

the homomorphism property is preserved. This fixes the representative of $\mu \in \operatorname{PSL}_{2}(\mathbb{R})$ to be

$$
V(\mu)=\exp \left(\lambda_{-1} \ell_{-1}\right) \exp \left(\lambda_{0} \ell_{0}\right) \exp \left(\lambda_{1} \ell_{1}\right),
$$

where the $\lambda_{k}$ 's are the ones corresponding to the group element $\mu^{-1}$.

\subsection{Parameters of the representation}

In order to derive the relation between the $\lambda_{k}$ 's and $\mu^{-1}$, we note that by (2.6)

$$
\exp \left(\lambda_{-1} \ell_{-1}\right) \exp \left(\lambda_{0} \ell_{0}\right) \exp \left(\lambda_{1} \ell_{1}\right) z=\frac{z+\lambda_{-1}}{-\lambda_{1} z+e^{-\lambda_{0}}-\lambda_{-1} \lambda_{1}}=\frac{A z+B}{C z+D} .
$$

Since $A D-B C=1$, we have

$$
D= \pm e^{\lambda_{0} / 2}\left(e^{-\lambda_{0}}-\lambda_{-1} \lambda_{1}\right)
$$

so that

$$
\lambda_{-1}=\frac{B}{A}, \quad e^{\lambda_{0}}=A^{2}, \quad \lambda_{1}=-\frac{C}{A},
$$

and

$$
\frac{A z+B}{C z+D}=\exp \left(\frac{B}{A} \ell_{-1}\right) \exp \left(2 \ln (A) \ell_{0}\right) \exp \left(-\frac{C}{A} \ell_{1}\right) z
$$

Since a global sign is irrelevant for $\mathrm{PSL}_{2}(\mathbb{R})$ matrices, we can choose the + sign on the right hand side of (2.10), so that

$$
\left(\begin{array}{ll}
A & B \\
C & D
\end{array}\right)=\left(\begin{array}{cc}
e^{\lambda_{0} / 2} & \lambda_{-1} e^{\lambda_{0} / 2} \\
-\lambda_{1} e^{\lambda_{0} / 2} & e^{-\lambda_{0} / 2}-\lambda_{-1} \lambda_{1} e^{\lambda_{0} / 2}
\end{array}\right)
$$


The above decomposition holds when $A \neq 0$. Group elements not attainable by the decomposition (2.9) can be reached by alternative decompositions. A decomposition which holds when $D \neq 0$ is the reversed one

$$
\exp \left(\lambda_{1} \ell_{1}\right) \exp \left(\lambda_{0} \ell_{0}\right) \exp \left(\lambda_{-1} \ell_{-1}\right) z=\frac{\left(e^{\lambda_{0}}-\lambda_{-1} \lambda_{1}\right) z+\lambda_{-1}}{-\lambda_{1} z+1}=\frac{A z+B}{C z+D} .
$$

Actually,

$$
A= \pm e^{-\lambda_{0} / 2}\left(e^{\lambda_{0}}-\lambda_{-1} \lambda_{1}\right)
$$

so that

$$
\lambda_{-1}=\frac{B}{D}, \quad e^{\lambda_{0}}=D^{-2}, \quad \lambda_{1}=-\frac{C}{D},
$$

and

$$
\frac{A z+B}{C z+D}=\exp \left(-\frac{C}{D} \ell_{1}\right) \exp \left(-2 \ln (D) \ell_{0}\right) \exp \left(\frac{B}{D} \ell_{-1}\right) z
$$

Choosing the + sign on the right hand side of (2.14), we have

$$
\left(\begin{array}{ll}
A & B \\
C & D
\end{array}\right)=\left(\begin{array}{cc}
e^{\lambda_{0} / 2}-\lambda_{-1} \lambda_{1} e^{-\lambda_{0} / 2} & -\lambda_{-1} e^{-\lambda_{0} / 2} \\
\lambda_{-1} e^{-\lambda_{0} / 2} & e^{-\lambda_{0} / 2}
\end{array}\right) .
$$

Finally, if both $A$ and $D$ vanish, we can choose

$$
\exp \left(\lambda_{-1} \ell_{-1}\right) \exp \left(\lambda_{-1}^{-1} \ell_{1}\right) \exp \left(\lambda_{-1} \ell_{-1}\right) z=-\frac{\lambda_{-1}^{2}}{z},
$$

so that

$$
B=-C^{-1}= \pm \lambda_{-1},
$$

where the sign ambiguity reflects the fact that this operator coincides with the one associated to the inverse matrix.

In the following we will need the operator version of eq. (2.8), that is

$$
V(\mu) f(z) V\left(\mu^{-1}\right)=f\left(\mu^{-1} z\right)
$$

Note that the relation (2.5) is represented by ${ }^{2}$

$$
V\left[\prod_{j=1}^{g}\left(\gamma_{2 j-1} \gamma_{2 j} \gamma_{2 j-1}^{-1} \gamma_{2 j}^{-1}\right)\right]=\prod_{j=1}^{g}\left(V_{2 j-1} V_{2 j} V_{2 j-1}^{-1} V_{2 j}^{-1}\right)=I,
$$

where we restricted to the hyperbolic case and

$$
V_{k} \equiv V\left(\gamma_{k}\right)
$$

\footnotetext{
${ }^{2}$ We will use $\mu$ and $\nu$ to denote generic elements of $\operatorname{PSL}_{2}(\mathbb{R})$, while $\beta$ and $\gamma$ will denote elements of the uniformizing group $\Gamma \subset \mathrm{PSL}_{2}(\mathbb{R})$.
} 


\section{Parameterization of $\mathrm{SL}_{2}(\mathbb{R})$ by the closed forms of the $\mathrm{BCH}$ formula}

We have seen that in order to express an element of $\mathrm{SL}_{2}(\mathbb{R})$ in the form

$$
\exp \left(\lambda_{i} \ell_{i}\right) \exp \left(\lambda_{j} \ell_{j}\right) \exp \left(\lambda_{k} \ell_{k}\right)
$$

one has to distinguish three different cases, namely

$$
A \neq 0, \quad D \neq 0, \quad A=D=0 .
$$

On the other hand, to find the covariant form of the conformal group, we must express (3.1) in the form

$$
\exp \left(\lambda_{i} \ell_{i}\right) \exp \left(\lambda_{j} \ell_{j}\right) \exp \left(\lambda_{k} \ell_{k}\right)=\exp \left(\mu_{1} \ell_{1}+\mu_{2} \ell_{2}+\mu_{3} \ell_{3}\right)
$$

for any choice of $i, j$ and $k$. Very recently, have been derived new closed forms for the BakerCampbell-Hausdorff (BCH) formula that include, as a particular case, the problem (3.3). Such a solution also solves the previous question, namely the one of writing down a unique expression, in the form on the right hand side of (3.3), holding simultaneously for the three distinguished cases (3.2). The reason is that any group element can be always expressed in the form on the right hand side of (3.3). Note that the unique exception would be if the trace of the corresponding matrix is -2 in the case when such a matrix is non-diagonalizable. On the other hand, we are interested in the linear fractional transformations where there is no any problem. The expression on the right of (3.3) is quite general. This has a deep consequence since it means that the covariantization of the conformal group is always definite. Let us further illustrate such a point, by considering $X, Y$ and $Z$, elements of an arbitrary Lie algebra $\mathfrak{g}$. Suppose that it has been found a finite linear combination $W$ of the generators of $\mathfrak{g}$ such that

$$
\exp (X) \exp (Y) \exp (Z)=\exp (W) .
$$

For any suitable norm, the power expansion of $\exp (X) \exp (Y) \exp (Z)$ converges on all $\mathbb{C}$, except in the points where one or more of the norms $\|X\|,\|Y\|$ and $\|Z\|$ are singular. It follows that if (3.4) holds in a neighborhood of the identity, then it should hold in a wider region with respect to the one related to the expansion of $\ln (\exp (X) \exp (Y) \exp (Z))$. Of course, this is related to the possible singularities of the norm of $W$. Remarkably, this never happens for $\mathrm{PSL}_{2}(\mathbb{C})$ matrices [4].

The problem of finding the closed form of $W$ in (3.4), has been recently considered in [4] where it has been introduced an algorithm that solves the $\mathrm{BCH}$ problem for a wide class of cases. In [5] it has been shown that there are 13 types of commutator algebras admitting such closed forms of the BCH formula. Furthermore, it turns out that the algorithm includes all the semisimple complex Lie algebras [6], as they correspond to particular cases of commutator algebras of type $1 c-i$, type 4 and type 5 . It turns out that $\mathrm{sl}_{2}(\mathbb{R})$ corresponds to a particular subtype of the type 4 commutator algebras.

Let us shortly show the main steps of the algorithm. In [7] Van-Brunt and Visser obtained a remarkable relation providing the closed form of the $\mathrm{BCH}$ formula in important cases. If $X, Y \in \mathfrak{g}$ satisfy the commutation relations

$$
[X, Y]=u X+v Y+c I,
$$


with $I$ a central element and $u, v, c$, complex parameters, then [7]

$$
\exp (X) \exp (Y)=\exp (X+Y+f(u, v)[X, Y])
$$

where $f(u, v)$ is the symmetric function

$$
f(u, v)=\frac{(u-v) e^{u+v}-\left(u e^{u}-v e^{v}\right)}{u v\left(e^{u}-e^{v}\right)} .
$$

Such a result generalizes to a wider class of cases by exploiting the associativity of the $\mathrm{BCH}$ formula and implementing the Jacobi identity [4]. Consider the identity

$$
\exp (X) \exp (Y) \exp (Z)=(\exp (X) \exp (\alpha Y))(\exp (\beta Y) \exp (Z))
$$

where

$$
\alpha+\beta=1 .
$$

If

$$
[X, Y]=u X+v Y+c I, \quad[Y, Z]=w Y+z Z+d I
$$

then, by (3.6),

$$
\exp (X) \exp (\alpha Y)=\exp (\tilde{X}), \quad \exp (\beta Y) \exp (Z)=\exp (\tilde{Y}),
$$

with

$$
\begin{aligned}
\tilde{X} & :=g_{\alpha}(u, v) X+h_{\alpha}(u, v) Y+l_{\alpha}(u, v) c I, \\
\tilde{Y} & :=h_{\beta}(z, w) Y+g_{\beta}(z, w) Z+l_{\beta}(z, w) d I,
\end{aligned}
$$

where

$$
\begin{aligned}
g_{\alpha}(u, v) & :=1+\alpha u f(\alpha u, v), \\
h_{\alpha}(u, v) & :=\alpha(1+v f(\alpha u, v)), \\
l_{\alpha}(u, v) & :=\alpha f(\alpha u, v) .
\end{aligned}
$$

This solves the BCH problem since, by (3.5), (3.6), (3.8) and (3.10), it follows that imposing

$$
[\tilde{X}, \tilde{Y}]=\tilde{u} \tilde{X}+\tilde{v} \tilde{Y}+\tilde{c} I
$$

that fixes $\alpha, \tilde{u}, \tilde{v}$ and $\tilde{c}$, gives

$$
\exp (X) \exp (Y) \exp (Z)=\exp (\tilde{X}) \exp (\tilde{Y})=\exp (\tilde{X}+\tilde{Y}+f(\tilde{u}, \tilde{v})[\tilde{X}, \tilde{Y}])
$$

Note that, consistently with the Jacobi identity

$$
[X,[Y, Z]]+[Y,[Z, X]]+[Z,[X, Y]]=0
$$

$[X, Z]$ may contain also $Y$

$$
[X, Z]=m X+n Y+p Z+e I
$$


The Jacobi identity constrains $e, m, n$ and $p$ by a linear system. Furthermore, note that setting $Y=\lambda_{0} Q$ and $\lambda_{-}:=\lambda_{0} \alpha, \lambda_{+}:=\lambda_{0} \beta$, eq. (3.8) implies, as a particular case,

$$
\exp (X) \exp (Z)=\lim _{\lambda_{0} \rightarrow 0} \exp (X) \exp \left(\lambda_{-} Q\right) \exp \left(\lambda_{+} Q\right) \exp (Z)
$$

explicitly showing that the algorithm solves also the $\mathrm{BCH}$ problem for $\exp (X) \exp (Z)$, in some of the cases when $[[X, Z], X]$ and $[[X, Z], Z]$ do not vanish.

In [4], as a particular case of the Virasoro algebra, it has been shown that the closed form of the $\mathrm{BCH}$ formula in the case of $\mathrm{sl}_{2}(\mathbb{C})$ is

$$
\begin{aligned}
\exp \left(\lambda_{-1} \ell_{-1}\right) & \exp \left(\lambda_{0} \ell_{0}\right) \exp \left(\lambda_{1} \ell_{1}\right) \\
& =\exp \left\{\frac{\lambda_{+}-\lambda_{-}}{e^{-\lambda_{-}}-e^{-\lambda_{+}}}\left[\lambda_{-1} \ell_{-1}+\left(2-e^{-\lambda_{+}}-e^{-\lambda_{-}}\right) \ell_{0}+\lambda_{1} \ell_{1}\right]\right\}
\end{aligned}
$$

where

$$
e^{-\lambda_{ \pm}}=\frac{1+e^{-\lambda_{0}}-\lambda_{-1} \lambda_{1} \pm \sqrt{\left(1+e^{-\lambda_{0}}-\lambda_{-1} \lambda_{1}\right)^{2}-4 e^{-\lambda_{0}}}}{2} .
$$

Note that, as explained above, since the $\ell_{k}$ 's are the differential representation of $\operatorname{sl}_{2}(\mathbb{R})$, it follows that the corresponding Möbius transformations are the ones associated to the inverse matrix. In fact, one may check that the correspondence between the $\lambda_{k}$ 's and the matrix elements given in eqs. $(2.10)(2.11)$, is the same of the one in [4] after the change

$$
\left(\begin{array}{ll}
A & B \\
C & D
\end{array}\right) \quad \longrightarrow \quad\left(\begin{array}{cc}
D & -B \\
-C & A
\end{array}\right) \text {. }
$$

\section{Setting the problem}

In the next sections we will construct the projective unitary representation of the fundamental group $\pi_{1}(\Sigma)$ by means of differential operators $\mathcal{U}_{k}$ acting on the Hilbert space $L^{2}(\mathbb{H})$. This will lead to a relation for such operators which has the form of (2.5) except that the identity on the right hand side is multiplied by a phase. In the hyperbolic case we will have

$$
\prod_{k=1}^{g} \mathcal{U}_{2 k-1} \mathcal{U}_{2 k} \mathcal{U}_{2 k-1}^{-1} \mathcal{U}_{2 k}^{-1}=e^{2 \pi i \theta} I
$$

As a first step, here we consider the unitarity problem. In higher genus we will see the appearance of several new structures. For example, a distinguished feature concerns the combination of differential operators that one may use to construct the unitary operators. While on the torus the exponentials $e^{\partial_{x_{1}}}$ and $e^{\partial_{x_{2}}}$ both appear separately, for $g>1$ the possible operators are restricted to a specific combination of $\partial_{1} \equiv \partial_{x_{1}}$ and $\partial_{2} \equiv \partial_{x_{2}}$.

In this section we will also reconsider the formulation of the noncommutative torus and, in particular, the way the phase $e^{2 \pi i \theta}$ in (1.1) is obtained. The aim is to learn from the torus as much as possible, in order to reformulate the derivation in a way which extracts the features of the construction without referring to the specifics of the torus. 
The fact that the fundamental group in $g>1$ is nonabelian implies that, in order to determine the phase in (4.1), we cannot use the reduced $\mathrm{BCH}$ formula

$$
e^{A} e^{B}=e^{[A, B]} e^{B} e^{A}
$$

which holds when $A$ and $B$ commute with $[A, B]$. For $g=1$ the associated differential generators commute, i.e. $\left[\partial_{1}, \partial_{2}\right]=0$, so that it makes sense to use (4.2) to evaluate the phase coming from the (constant) commutator of the covariant derivatives. For $g>1$, a computation of the phase by means of the complete $\mathrm{BCH}$ formula would involve quantities which are a covariantization of the already noncommuting operators such as the generators of $\mathrm{sl}_{2}(\mathbb{R})$. However, this is not only a technical difficulty, rather we actually still do not know which structures the covariantization of the generators of $\operatorname{sl}_{2}(\mathbb{R})$ may have. As we said, reformulating the case of the torus in a different language will suggest its natural higher genus generalization, without using the complete $\mathrm{BCH}$ formula. In particular, it will shed lights on the covariantization procedure. As we will see, the result is deeply related to the geometry of Riemann surfaces. In particular, constructing the unitary operators that will projectively represent $\pi_{1}(\Sigma)$, will bring us to a problem that can be seen as the one of simultaneous covariantization. Essentially, this is the problem of finding in higher genus the covariant version

$$
\mathcal{O}_{A}=F \mathcal{O} F^{-1}
$$

of a given operator $\mathcal{O}$ in such a way that its adjoint has the form $\mathcal{O}_{A}^{\dagger}=F \tilde{\mathcal{O}} F^{-1}$, with $\tilde{\mathcal{O}}$ independent of the connection $A$ and $F$ a functional of $A$.

\subsection{A first screening}

Mimicking the case of $g=1$, where each one of the two operators $\mathcal{U}_{1}$ and $\mathcal{U}_{2}$ are expressed in terms of real coordinates $x \equiv x_{1}$ and $y \equiv x_{2}$ respectively, one expects that the building blocks for the solution to the quotient conditions in $g>1$ have two possible forms, either

$$
\exp \left(\mathcal{L}_{n}-\mathcal{L}_{n}^{\dagger}\right)
$$

or

$$
\exp \left(i\left(\mathcal{L}_{n}+\mathcal{L}_{n}^{\dagger}\right)\right)
$$

where the $\mathcal{L}_{n}$ 's are some covariantized $\mathrm{sl}_{2}(\mathbb{R})$ operators to be determined. Such operators should be the generalization to the case of the three generators of $\operatorname{sl}_{2}(\mathbb{R})$ of the covariant derivative. Since in the case of the torus the relevant phase is expressed by means of the commutator between covariant derivatives, and considering that $\mathcal{L}_{n}$ contains $\partial_{z}=\partial_{x}-i \partial_{y}$, one may expect that, in the case of higher genus Riemann surfaces, both $\exp \left(\mathcal{L}_{n}-\mathcal{L}_{n}^{\dagger}\right)$ and $\exp \left(i\left(\mathcal{L}_{n}+\mathcal{L}_{n}^{\dagger}\right)\right)$ appear. However, while the exponentials $\exp \left(\partial_{x}\right)$ and $\exp \left(\partial_{y}\right)$ generate translations, that map $\mathbb{C}$, the universal covering of the torus, to itself, the operators $\exp \left(\mathcal{L}_{n}-\mathcal{L}_{n}^{\dagger}\right)$ and $\exp \left(i\left(\mathcal{L}_{n}+\mathcal{L}_{n}^{\dagger}\right)\right)$ should generate $\mathrm{PSL}_{2}(\mathbb{R})$ transformations. On the other hand, $\exp \left(i\left(\mathcal{L}_{n}+\mathcal{L}_{n}^{\dagger}\right)\right)$ cannot generate real Möbius transformations, so that we should discard $\exp \left(i\left(\mathcal{L}_{n}+\mathcal{L}_{n}^{\dagger}\right)\right)$ and restrict to $\exp \left(\mathcal{L}_{n}-\mathcal{L}_{n}^{\dagger}\right)$ only. This fact is strictly related to the nonabelian nature of the group $\pi_{1}(\Sigma)$ which, in turn, is related to the condition $\Im(z)>0$ defining $\mathbb{H}$. The latter reflects the fact that the translation operator along 
the imaginary axis $\exp \left(\partial_{y}\right)$ alone does not belong to the automorphisms group of $\mathbb{H}$. Since among the translation operators $\exp \left(\partial_{x}\right)$ and $\exp \left(\partial_{y}\right)$ only the former is allowed, we see that comparing $\exp \left(\mathcal{L}_{n}-\mathcal{L}_{n}^{\dagger}\right)$ with $\exp \left(\partial_{x}\right)=\exp \left(\left(\partial_{z}+\partial_{\bar{z}}\right) / 2\right)$ one should expect that $\mathcal{L}_{n}^{\dagger}$ corresponds to $-\overline{\mathcal{L}}_{n}$. We will see that a slightly modified version of this holds.

Finding the $\mathcal{L}_{n}$ 's is a problem closely related to the one of deriving the central extension for the Fuchsian group without using the $\mathrm{BCH}$ formula. Since the $\mathcal{L}_{n}$ 's are covariant versions of the three generators of $\mathrm{sl}_{2}(\mathbb{R})$, in general the nested commutators

$$
\left[\mathcal{L}_{j_{1}},\left[\mathcal{L}_{j_{2}},\left[\cdots\left[\mathcal{L}_{j_{n-1}}, \mathcal{L}_{j_{n}}\right]\right] \cdots\right]\right]
$$

should be difficult to treat, so that, apparently, one should use the complete version of the BCH formula. Nevertheless, since we will succeed in finding the central extension of the uniformizing group, this implies that the same result should be obtained by using the complete $\mathrm{BCH}$ formula. Therefore, in spite of (4.4), the structure of the $\mathrm{sl}_{2}(\mathbb{R})$ implies a simplification. In particular, determining the $c_{j_{1} \ldots j_{n}}$ in

$$
\sum_{l, m=-1}^{1} c_{j_{1} \ldots j_{n}}\left[\mathcal{L}_{l}, \mathcal{L}_{m}\right]=\left[\mathcal{L}_{j_{1}},\left[\mathcal{L}_{j_{2}},\left[\cdots\left[\mathcal{L}_{j_{n-1}}, \mathcal{L}_{j_{n}}\right]\right] \cdots\right]\right]
$$

should reveal a considerable simplification of the complete $\mathrm{BCH}$ formula for the case at hand.

\subsection{An alternative to the $\mathrm{BCH}$ formula on the torus}

Here we revisit the covariantization of the translation operators. These are naturally associated to the tessellation of the plane, so that their covariantization leads to consider the algebra of the noncommutative torus. For sake of simplicity, we consider the covariantization of translations operators along the Cartesian coordinates of $\mathbb{R}^{2}$, denoted by $x_{1}$ and $x_{2}$. These operators are naturally associated to orthogonal tori. The investigation will lead to the computation of the phase in eq. (1.1) without using the BCH formula. This alternative will indicate the way to covariantize the $\mathrm{PSL}_{2}(\mathbb{R})$ operators, therefore providing the extension of our analysis to $g>1$, where the direct use of the $\mathrm{BCH}$ formula is, apparently, inextricable.

Let us consider the connection

$$
A=A_{1} d x_{1}+A_{2} d x_{2},
$$

and the operators

$$
\mathcal{U}_{k}=\exp \left(\lambda_{k}\left(\partial_{k}+i A_{k}\right)\right),
$$

$k=1,2$, where $\lambda_{k} \in \mathbb{R}$. Given two operators $A$ and $B$, and a function $f(B)$ satisfying suitable properties, we have

$$
A f(B) A^{-1}=f\left(A B A^{-1}\right) .
$$

We define the functions $F_{k}\left(x_{1}, x_{2}\right), k=1,2$, by

$$
\mathcal{U}_{k}=F_{k} \exp \left(\lambda_{k} \partial_{k}\right) F_{k}^{-1},
$$


that compared with (4.6) yields

$$
\left(\partial_{k}+i A_{k}\right) F_{k}=0
$$

The solution of this equation is

$$
F_{1}\left(x_{1}, x_{2}\right)=\exp \left(-i \int_{\left(x_{1}^{0}, x_{2}\right)}^{\left(x_{1}, x_{2}\right)} d x A_{1}\left(x, x_{2}\right)\right),
$$

where the contour integral is with $x_{2}$ fixed. Similarly

$$
F_{2}\left(x_{1}, x_{2}\right)=\exp \left(-i \int_{\left(x_{1}, x_{2}^{0}\right)}^{\left(x_{1}, x_{2}\right)} d x A_{2}\left(x_{1}, x\right)\right)
$$

where the contour integral is with $x_{1}$ fixed. In the following we will use the notation

$$
F_{k}=\exp \left(-i \int_{x_{k}^{0}}^{x_{k}} d a_{k} A_{k}\right)
$$

where in the integrand one has $A_{1}\left(a_{1}, x_{2}\right)$ if $k=1$ and $A_{2}\left(x_{1}, a_{2}\right)$ if $k=2$. There is an observation that simplifies considerably the construction. The key point is that since in the two contour integrals either $x_{1}$ or $x_{2}$ are fixed, so that either $d x_{1}=0$ or $d x_{2}=0$, it follows that both the integrands, $d a_{1} A_{1}$ and $d a_{2} A_{2}$, can be replaced by the full connection $A=A_{1} d x_{1}+A_{2} d x_{2}$. Therefore,

$$
F_{k}=\exp \left(-i \int_{x_{k}^{0}}^{x_{k}} A\right) .
$$

Since $\exp \left(\lambda_{k} \partial_{k}\right)$ is the translation operator, we have

$$
\begin{aligned}
\mathcal{U}_{k} & =\exp \left(-i \int_{x_{k}^{0}}^{x_{k}} A\right) \exp \left(\lambda_{k} \partial_{k}\right) \exp \left(i \int_{x_{k}^{0}}^{x_{k}} A\right) \\
& =\exp \left(i \int_{x_{k}}^{x_{k}+\lambda_{k}} A\right) \exp \left(\lambda_{k} \partial_{k}\right) .
\end{aligned}
$$

This allows for a very geometric derivation of the phase in (1.1).

Since we are investigating the covariantization of $\partial_{x_{1}}$ and $\partial_{x_{2}}$, we consider tori whose fundamental domain $\mathcal{F}$ is a rectangle. Denote by $\lambda_{1}$ and $\lambda_{2}$ its base and height respectively. By (4.7) and Stokes' theorem

$$
\begin{aligned}
\mathcal{U}_{1} \mathcal{U}_{2} \mathcal{U}_{1}^{-1} \mathcal{U}_{2}^{-1}=\exp \left[i \int_{\left(x_{1}, x_{2}\right)}^{\left(x_{1}+\lambda_{1}, x_{2}\right)} A+i \int_{\left(x_{1}+\lambda_{1}, x_{2}\right)}^{\left(x_{1}+\lambda_{1}, x_{2}+\lambda_{2}\right)} A\right. \\
\left.+i \int_{\left(x_{1}+\lambda_{1}, x_{2}+\lambda_{2}\right)}^{\left(x_{1}, x_{2}+\lambda_{2}\right)} A+i \int_{\left(x_{1}, x_{2}+\lambda_{2}\right)}^{\left(x_{1}, x_{2}\right)} A\right] \\
=\exp \left(i \oint_{\partial \mathcal{F}} A\right)=\exp \left(i \int_{\mathcal{F}} F\right),
\end{aligned}
$$

where $F$ is the curvature of $A$

$$
F=d A=\left(\partial_{1} A_{2}-\partial_{2} A_{1}\right) d x_{1} \wedge d x_{2}=F_{12} d x_{1} \wedge d x_{2} .
$$


The above shows that the phase does not equal the curvature $F$. The fact that our derivation and the one made with the reduced $\mathrm{BCH}$ formula (4.2) coincide, is due to the fact that a possible solution is given by a constant $F_{12}$. A possible choice to get a constant phase is to set $F_{12}=2 \pi \theta / \lambda_{1} \lambda_{2}$ that corresponds to

$$
A_{1}=-\pi \frac{\theta}{\lambda_{1} \lambda_{2}} x_{2}, \quad A_{2}=\pi \frac{\theta}{\lambda_{1} \lambda_{2}} x_{1} .
$$

that by (4.2) and (4.6) gives

$$
\begin{aligned}
\exp \left(\lambda _ { 1 } \left(\partial_{1}\right.\right. & \left.\left.+i A_{1}\right)\right) \exp \left(\lambda_{2}\left(\partial_{2}+i A_{2}\right)\right) \\
& =\exp \left(\lambda_{1} \lambda_{2}\left[\partial_{1}+i A_{1}, \partial_{2}+i A_{2}\right]\right) \exp \left(\lambda_{2}\left(\partial_{2}+i A_{2}\right)\right) \exp \left(\lambda_{1}\left(\partial_{1}+i A_{1}\right)\right),
\end{aligned}
$$

that is (1.1). However, only in the case in which $F_{12}$ is constant does one have

$$
\int_{\mathcal{F}} F=\lambda_{1} \lambda_{2} F_{12}
$$

Let us now show why apparently the constant curvature connection is the unique solution. Let us add the suffix $x_{1} x_{2}$ to $\mathcal{F}$ in order to indicate its dependence on the base-point. Also, note that $\mathcal{F}$ is univocally determined by $x_{1}$ and $x_{2}$. We need to show that the integral of $F$ on $\mathcal{F}_{x_{1} x_{2}}$ is independent of the point $\left(x_{1}, x_{2}\right)$. That is

$$
\int_{\mathcal{F}_{x_{1} x_{2}}} F=\int_{\mathcal{F}_{x_{1}^{\prime} x_{2}^{\prime}}} F,
$$

for any $\left(x_{1}^{\prime}, x_{2}^{\prime}\right) \in \mathbb{R}^{2}$. Any point in $\mathbb{R}^{2}$ can be obtained by a translation

$$
\left(x_{1}, x_{2}\right) \rightarrow\left(x_{1}^{\prime}, x_{2}^{\prime}\right)=\mu\left(x_{1}, x_{2}\right) \equiv\left(x_{1}+b_{1}, x_{2}+b_{2}\right) .
$$

Let us apply the translation $\mu$ to the entire fundamental domain and denote it by $\mu \mathcal{F}_{x_{1} x_{2}}$. Since $\mathcal{F}_{x_{1}^{\prime} x_{2}^{\prime}}=\mu \mathcal{F}_{x_{1} x_{2}}$, we have

$$
\int_{\mathcal{F}_{x_{1}^{\prime} x_{2}^{\prime}}} F=\int_{\mathcal{F}_{x_{1} x_{2}}} \mu^{\star} F,
$$

so that eq. (4.11) is satisfied only if

$$
\int_{\mathcal{F}_{x_{1} x_{2}}}\left(\mu^{\star} F-F\right)=0 .
$$

This fixes $F$ to be a constant two-form, up to a non-constant contribution with vanishing surface integral. This implies a hidden invariance that we consider below.

\subsection{The hidden invariance}

Let us still consider tori with rectangular fundamental domain. We now show that the above investigation also allows to find an additional invariance of the operators

$$
\mathcal{U}_{k}=\exp \left(\lambda_{k}\left(\partial_{k}+i A_{k}\right)\right)
$$


under a suitable transformation of the connection $A_{k}$. Such an invariance, which is not evident by analyzing the $\mathcal{U}_{k}$ 's expressed in the form $\exp \left(\lambda_{k}\left(\partial_{k}+i A_{k}\right)\right)$, is a consequence of the fact that $\exp \left(\lambda_{k} \partial_{k}\right)$ is a translation operator. By (4.7) we see that also $\mathcal{U}_{k}$ is a translation operator

$$
\mathcal{U}_{1} f\left(x_{1}, x_{2}\right)=f\left(x_{1}+\lambda_{1}, x_{2}\right) \mathcal{U}_{1}, \quad \mathcal{U}_{2} f\left(x_{1}, x_{2}\right)=f\left(x_{1}, x_{2}+\lambda_{2}\right) \mathcal{U}_{2} .
$$

Therefore, the $\mathcal{U}_{k}$ 's have the invariance property

$$
\begin{aligned}
h_{k} \mathcal{U}_{k} h_{k}^{-1} & =h_{k} \exp \left(-i \int_{x_{k}^{0}}^{x_{k}} A\right) \exp \left(\lambda_{k} \partial_{k}\right) \exp \left(i \int_{x_{k}^{0}}^{x_{k}} A\right) h_{k}^{-1} \\
& =\exp \left(i \int_{x_{k}}^{x_{k}+\lambda_{k}} A\right) \exp \left(\lambda_{k} \partial_{k}\right)=\mathcal{U}_{k},
\end{aligned}
$$

whenever the $h_{k}$ 's satisfy

$$
h_{1}\left(x_{1}, x_{2}\right) h_{1}^{-1}\left(x_{1}+\lambda_{1}, x_{2}\right)=1, \quad h_{2}\left(x_{1}, x_{2}\right) h_{2}^{-1}\left(x_{1}, x_{2}+\lambda_{2}\right)=1 .
$$

To preserve unitarity of $\mathcal{U}_{k}$, we require $\left|h_{k}\right|=1$. Thus, the transformation of the connection

$$
A_{k} \longrightarrow A_{k}+i \partial_{k} \ln h_{k}
$$

leaves $\mathcal{U}_{k}$ invariant.

In general (4.14) is not a gauge transformation, as each component transforms according to a different $h_{k}$. Consequently, under (4.14) the curvature $F$ transforms to

$$
\tilde{F}=F-i \partial_{1} \partial_{2} \ln \frac{h_{1}}{h_{2}} d x_{1} \wedge d x_{2},
$$

while the phase (4.8) remains invariant. This transformation can be restricted to one component of the connection only, that is we can choose either $h_{1}$ or $h_{2}$ to be a constant. Also, the operators $\mathcal{U}_{k}$ are not gauge invariant unless the gauge function is periodic up to a multiple of $2 \pi$. That is, by (4.13) it follows that $\mathcal{U}_{k}$ is invariant under the gauge transformation

$$
A \rightarrow A+d \chi
$$

if and only if

$$
\chi\left(x_{1}+\lambda_{1}, x_{2}\right)=\chi\left(x_{1}, x_{2}\right)+2 m \pi, \quad \chi\left(x_{1}, x_{2}+\lambda_{2}\right)=\chi\left(x_{1}, x_{2}\right)+2 m \pi,
$$

$m, n \in \mathbb{Z}$. Of course, this is consistent with the fact that the transformation (4.14) is not a gauge transformation unless $h_{1}=$ cnst $h_{2}$.

Above we saw how to remove the gauge connection $A$ from the covariant derivative in $\mathcal{U}_{k}$. The result is that the gauge connection appears integrated along straight lines, that is geodesics with respect to the flat metric, the right language to extend the construction to higher genus. Upon factorization, the connection is acted on by the inverse of the differential operator (integration). This shows a close relation between integrals of the connection along geodesics, i.e. $\int_{x_{k}}^{x_{k}+\lambda_{k}} A$, and covariantized operators. More precisely, inverting (4.7) yields

$$
\exp \left(i \int_{x_{k}}^{x_{k}+\lambda_{k}} A\right)=\exp \left(\lambda_{k}\left(\partial_{k}+i A_{k}\right)\right) \exp \left(-\lambda_{k} \partial_{k}\right) .
$$




\section{Unitary projective representations of the Fuchsian group}

\subsection{The problem of simultaneous covariantization}

In the following we will see that, as expected, in higher genus it is convenient to use complex coordinates $z$ and $\bar{z}$ rather than real ones $x$ and $y$. Some of the operators we will work with have the structure

$$
\exp \left(\nabla_{z}-\nabla_{z}^{\dagger}\right)
$$

which is unitary by construction. The operators $\nabla_{z}$ and $\nabla_{z}^{\dagger}$ will be some appropriate covariantization of $\partial_{z}$ and $\partial_{\bar{z}}$. Since we will also consider the covariantization of the $\mathrm{sl}_{2}(\mathbb{R})$ differential operators, the noncommutativity of the latter will naturally lead to the method described in the previous subsections, rather than to the reduced $\mathrm{BCH}$ formula (4.2).

The alternative derivation of the phase considered above may be applied to other cases if one can set ${ }^{3}$

$$
\exp \left(\nabla_{z}-\nabla_{z}^{\dagger}\right)=F \exp \left(\partial_{z}+\partial_{\bar{z}}\right) F^{-1},
$$

for some suitable function $F(z, \bar{z})$. The fact that we need such simultaneous covariantization, i.e., that we need to express both the covariant derivative and its adjoint as standard derivatives conjugate by the same function, has already been used in the case of the torus. In that case we considered the action of unitary operators on the $F_{k}$ rather than the reduced $\mathrm{BCH}$ formula. It is clear that if we had, for example, $\nabla_{z} \propto F \partial_{z} F^{-1}$ and $\nabla_{z}^{\dagger} \propto F^{-1} \partial_{\bar{z}} F$, then it would not be possible to extend the above method to $g>1$. In that case eq. (5.1) would not hold, and it would not be possible to get the phase on the right hand side of (4.1) by using the action of differential operators

$$
\exp \left(\partial_{z}+\partial_{\bar{z}}\right) F^{-1}(z, \bar{z})=F^{-1}(z+1, \bar{z}+1) \exp \left(\partial_{z}+\partial_{\bar{z}}\right) .
$$

As an example, one can consider differential operators acting on automorphic functions on the upper half-plane, or, what is equivalent, covariant operators acting on sections of line bundles on a Riemann surface $\Sigma .^{4}$ These operators can be seen as a sort of covariantization of $\partial_{z}$ and $\partial_{\bar{z}}$. More precisely, consider the metric tensor $g_{z \bar{z}}$, so that $d s^{2}=2 g_{z \bar{z}} d z d \bar{z}$. The covariant derivative acting on $K^{\lambda}$, where $K$ is the canonical line bundle on $\Sigma$, is

$$
\nabla_{z}^{\lambda}: K^{\lambda} \longrightarrow K^{\lambda+1}
$$

where

$$
\nabla_{z}^{\lambda} \psi=g_{z \bar{z}}^{\lambda} \partial_{z} g_{z \bar{z}}^{-\lambda} \psi=\left(\partial_{z}-\lambda \partial_{z} \ln g_{z \bar{z}}\right) \psi
$$

Formally, one can consider this as a suitable covariantization of $\partial_{z}$. The scalar product on $K^{\lambda}$ is

$$
\langle\phi \mid \psi\rangle=\int_{\Sigma} d \nu \sqrt{g}\left(g^{z \bar{z}}\right)^{\lambda} \bar{\phi} \psi,
$$

\footnotetext{
${ }^{3}$ The above cannot correspond to $F(z, \bar{z}) \exp \left(\partial_{z}-\partial_{\bar{z}}\right) F^{-1}(z, \bar{z})$, as $\exp \left(\partial_{z}-\partial_{\bar{z}}\right)$ would correspond to translations of $\Im(z)$ by an imaginary constant and cannot be unitary. On the torus we can also use $\exp \left(i\left(\nabla_{z}+\nabla_{z}^{\dagger}\right)\right)$. However, as seen in subsection 4.1, the nonabelian nature of $\pi_{1}(\Sigma)$ forbids the use of $i$.

${ }^{4}$ To simplify notation, we will use the same symbol $z$ to denote a coordinate both on $\mathbb{H}$ and $\Sigma$.
} 
where

$$
d \nu(z)=\frac{i}{2} d z \wedge d \bar{z}
$$

It follows that the adjoint of $\nabla_{z}^{\lambda}$

$$
\left(\nabla_{z}^{\lambda}\right)^{\dagger}: K^{\lambda+1} \longrightarrow K^{\lambda}
$$

is

$$
\left(\nabla_{z}^{\lambda}\right)^{\dagger} \psi=-g^{z \bar{z}} \partial_{\bar{z}} \psi
$$

In the literature there are also other examples of covariant differential operators on higher genus Riemann surfaces. For example one can consider covariant operators acting on sections of $K^{\mu} \otimes \bar{K}^{\nu}$, and then take the dual space to be sections of $K^{\rho} \otimes \bar{K}^{\sigma}$ for some $\rho$ and $\sigma$. In particular, for a suitable choice of the weights $\mu, \nu, \rho$ and $\sigma$, one can obtain covariant operators of the form $g_{z \bar{z}}^{\lambda} \partial_{z} g_{z \bar{z}}^{-\lambda}$, with adjoints $-g_{z \bar{z}}^{-\lambda} \partial_{\bar{z}} g_{z \bar{z}}^{\lambda}$ which, in a certain sense, exhibit more symmetry than (5.2) and (5.3). Nevertheless, also in this case we have $\partial_{z}$ conjugate by $g_{z \bar{z}}^{\lambda}$ and $g_{z \bar{z}}^{-\lambda}$, whereas $\partial_{\bar{z}}$ is conjugate in the reverse order, that is by $g_{z \bar{z}}^{-\lambda}$ and $g_{z \bar{z}}^{\lambda}$. Thus, an apparently unavoidable feature of covariant operators is that they never admit the simultaneous covariantization

$$
\nabla_{z}-\nabla_{z}^{\dagger}=F(z, \bar{z})\left(\partial_{z}+\partial_{\bar{z}}\right) F^{-1}(z, \bar{z})
$$

for some $F$. On the other hand, we have just seen that we need precisely the property that, given a covariant differential operator (in particular, an $\mathrm{sl}_{2}(\mathbb{R})$ differential operator) and its adjoint, both of them should be expressed as a non covariant operator conjugate by $F$ and $F^{-1}$ in the same order.

Let us further illustrate this point. In evaluating the adjoint operator, one performs an integration by parts and simultaneously takes the complex conjugate. Complex conjugation is the crucial point. In fact, if we now construct covariantized operators by conjugating them by $F$ and $F^{-1}$, with $F$ a phase, then complex conjugation corresponds to the inversion of $F$. Thus, the unique solution is to choose

$$
\bar{F}=F^{-1},
$$

and define

$$
\nabla_{z}=F \partial_{z} F^{-1}
$$

Note that the condition $|F|=1$ essentially follows also by requiring the unitarity of the operator in (5.1). Let us consider the scalar product

$$
\langle\phi \mid \psi\rangle=\int_{\mathbb{H}} d \nu \bar{\phi} \psi
$$

integrating by parts, by (5.5) we have

$$
\left.\langle\phi \mid \nabla \psi\rangle=\int_{\mathbb{H}} d \nu \bar{\phi} F \partial_{z} F^{-1} \psi=-\int_{\mathbb{H}} d \nu \overline{F \partial_{\bar{z}}\left(F^{-1} \phi\right.}\right) \psi=\left\langle\nabla_{z}^{\dagger} \phi \mid \psi\right\rangle,
$$


showing that the adjoint $\nabla_{z}^{\dagger}$ is constructed by conjugating $\partial_{\bar{z}}$ in the same way as $-\partial_{z}$ is conjugate to get $\nabla_{z}$

$$
\nabla_{z}^{\dagger}=-F \partial_{\bar{z}} F^{-1}
$$

Therefore, we found the way of covariantizing the derivatives as in (5.4). Later on we will apply the above method to the case of the $\mathrm{sl}_{2}(\mathbb{R})$ differential operators.

The above integration domain is $\mathbb{H}$ rather than the Riemann surface itself, as was the case previously. Our equations will be defined on the upper half-plane, as we are interested in constructing a projective unitary representation of the group $\pi_{1}(\Sigma)$ by means of operators acting on $L^{2}(\mathbb{H})$. In particular, the action of our operators will not be restricted to automorphic forms, which is the case when the equations are to be projected onto the Riemann surface. In this respect we now show that trying to perform a similar trick in the case of scalar products defined on a Riemann surface would lead to imaginary powers of the metric. Denoting by $\psi^{(\mu, \sigma)}$ a section of $K^{\mu} \otimes \bar{K}^{\sigma}$, we see that

$$
\int_{\Sigma} \overline{\phi^{(\rho, i \kappa)}} g_{z \bar{z}}^{i \kappa} \partial_{z} g_{z \bar{z}}^{-i \kappa} \psi^{(i \kappa, 1-\rho)}=-\int_{\Sigma} \overline{g_{z \bar{z}}^{i \kappa} \partial_{\bar{z}}\left(g_{z \bar{z}}^{-i \kappa} \phi^{(\rho, i \kappa)}\right)} \psi^{(i \kappa, 1-\rho)}
$$

where $\rho, \kappa$ are real numbers. ${ }^{5}$ The integrand in $(5.6)$ is a $(1,1)$-form, and the action of the derivatives is covariant, that is, they act on 0-differentials (e.g. $\partial_{z} g_{z \bar{z}}^{-i \kappa} \psi^{(i \kappa, 1-\rho)}$ ). Also, the complex conjugate of a $(\mu, \nu)$ differential is a $(\bar{\nu}, \bar{\mu})$ differential. ${ }^{6}$ Eq. (5.6) implies that the adjoint of

$$
\nabla_{z}=g_{z \bar{z}}^{i \kappa} \partial_{z} g_{z \bar{z}}^{-i \kappa}
$$

is

$$
\nabla_{z}^{\dagger}=-g_{z \bar{z}}^{i \kappa} \partial_{\bar{z}} g_{z \bar{z}}^{-i \kappa}
$$

As a consequence of the fact that both $\nabla_{z}$ and $\nabla_{z}^{\dagger}$ are obtained by conjugating $\partial_{z}$ and $-\partial_{\bar{z}}$ by $g_{z \bar{z}}^{i \kappa}$ and $g_{z \bar{z}}^{-i \kappa}$, we have that a function of any linear combination of $\nabla_{z}$ and of its adjoint has the property

$$
f\left(a \nabla_{z}+b \nabla_{z}^{\dagger}\right)=g_{z \bar{z}}^{i \kappa} f\left(a \partial_{z}+b \partial_{\bar{z}}\right) g_{z \bar{z}}^{-i \kappa} .
$$

The appearance of the phase ties together several mathematical aspects which have a physical meaning. In particular, considers the operators (5.7) and (5.8) on the upper half-plane endowed with the Poincaré metric

$$
d s^{2}=y^{-2}|d z|^{2}=2 g_{z \bar{z}}|d z|^{2}=e^{\varphi}|d z|^{2},
$$

then one would obtain $\nabla_{z}=y^{-2 i \kappa} \partial_{z} y^{2 i \kappa}$ and $\nabla_{z}^{\dagger}=-y^{-2 i \kappa} \partial_{\bar{z}} y^{2 i \kappa}$. In this respect, it is interesting to observe that the Poincaré Laplacian

$$
\Delta=-4 y^{2} \partial_{\bar{z}} \partial_{z}
$$

\footnotetext{
${ }^{5}$ The construction can be generalized to the case in which the weight $\rho$ is a complex number. The only difference consists in replacing $\psi^{(i \kappa, 1-\rho)}$ by $\psi^{(i \kappa, 1-\bar{\rho})}$. One may also consider replacing $i \lambda$ by a complex number $\mu$, however in such a case $g_{z \bar{z}}^{\mu}$ would not be a phase and simultaneous covariantization would not be possible.

${ }^{6}$ Differentials have been studied in the literature with real $[9,10]$ and complex weights [11].
} 
satisfies the equation

$$
\Delta y^{\frac{1}{2}+i \kappa}=\lambda_{\kappa} y^{\frac{1}{2}+i \kappa},
$$

where the eigenvalues are

$$
\lambda_{\kappa}=\frac{1}{4}+\kappa^{2} .
$$

The problem of simultaneous covariantization led us to introduce imaginary powers of the metric. In turn, the associated Laplacian has eigenfuctions corresponding to complex powers of such a metric. On the other hand, the appearance of this complex power lies at the heart of the mass gap $1 / 4$ in (5.9) which never appears in flat spaces such as in the case of the torus. Thus, there is a strict relationship between the noncommutativity of the fundamental group $\pi_{1}(\Sigma)$, the structures derived from imposing the simultaneous covariantization, and the structure of the Laplacian eigenvalues themselves. The mass gap $1 / 4$ is in fact a sort of regularization induced by the negative curvature (which in turn is related to the nonabelian nature of $\pi_{1}(\Sigma)$ ). In this context, it is worth mentioning that in [12] curvature brings the infrared and ultraviolet behavior of QCD under analytic control without any conflict with gauge invariance.

\subsection{The unitary covariantized operators}

Set

$$
e_{n}(z)=z^{n+1},
$$

$n=-1,0,1$, and define the operators

$$
L_{n}=e_{n}^{1 / 2} \partial_{z} e_{n}^{1 / 2}=e_{n}^{-1 / 2} \ell_{n} e_{n}^{1 / 2}=e_{n}\left(\partial_{z}+\partial_{z} \ln e_{n}^{1 / 2}\right),
$$

that is

$$
L_{1}=z^{2} \partial_{z}+z=\ell_{1}+z, \quad L_{0}=z \partial_{z}+\frac{1}{2}=\ell_{0}+\frac{1}{2}, \quad L_{-1}=\partial_{z}=\ell_{-1} .
$$

This deformation of the $\ell_{n}$ has no effect on the algebra, that is

$$
\left[L_{m}, L_{n}\right]=(n-m) L_{m+n} .
$$

Furthermore

$$
\left[L_{n}, f\right]=z^{n+1} \partial_{z} f .
$$

Let us define the covariantized operators

$$
\mathcal{L}_{n}^{(F)}=F L_{n} F^{-1}=F e_{n}^{1 / 2} \partial_{z} e_{n}^{1 / 2} F^{-1}=e_{n}\left[\partial_{z}+\partial_{z} \ln \left(e_{n}^{1 / 2} F^{-1}\right)\right],
$$

where $F(z, \bar{z})$ is an arbitrary function of unitary modulo

$$
|F|=1 \text {. }
$$

Since the $\mathcal{L}_{n}^{(F)}$ and $L_{n}$ differ by a conjugation, it follows that the $\mathcal{L}_{n}^{(F)}$ satisfy the same algebra of the $L_{n}$

$$
\left[\mathcal{L}_{m}^{(F)}, \mathcal{L}_{n}^{(F)}\right]=(n-m) \mathcal{L}_{m+n}^{(F)}
$$


Introducing the scalar product on $L^{2}(\mathbb{H})$

$$
\langle\phi \mid \psi\rangle=\int_{\mathbb{H}} d \nu \bar{\phi} \psi
$$

we see that an integration by parts leads to

$$
\left\langle\phi \mid \mathcal{L}_{n}^{(F)} \psi\right\rangle=-\int_{\mathbb{H}} d \nu \overline{F \overline{e_{n}^{1 / 2}} \partial_{\bar{z}}\left(\overline{e_{n}^{1 / 2}} F^{-1} \phi\right)} \psi=\left\langle\mathcal{L}_{n}^{(F) \dagger} \phi \mid \psi\right\rangle,
$$

that is the adjoint of $\mathcal{L}_{n}^{(F)}$

$$
\mathcal{L}_{n}^{(F) \dagger}=-F \overline{e_{n}^{1 / 2}} \partial_{\bar{z}} \overline{e_{n}^{1 / 2}} F^{-1}=-\overline{\mathcal{L}}_{n}^{\left(F^{-1}\right)} .
$$

Comparing

$$
\mathcal{L}_{n}^{(F)^{\dagger}}=\left(F e_{n}^{1 / 2} \partial_{z} e_{n}^{1 / 2} F^{-1}\right)^{\dagger}=F \overline{e_{n}^{1 / 2}} \partial_{z}^{\dagger} e_{n}^{\overline{1 / 2}} F^{-1},
$$

with (5.10) one obtains

$$
\ell_{n}^{\dagger}=-\bar{e}_{n}^{-1} \bar{\ell}_{n} \bar{e}_{n}
$$

In the case $n=-1$ we have $\partial_{z}^{\dagger}=-\partial_{\bar{z}}$, so that, since $\partial_{z}=\left(\partial_{x}-i \partial_{y}\right) / 2$, we see that the construction reproduces the usual adjoint operation in the case of $\partial_{x}$ and $\partial_{y}$.

The basic property of the adjoint $\mathcal{L}_{n}^{(F) \dagger}$ is that it is obtained by conjugating $-\bar{L}_{n}$ with $F$ and $F^{-1}$. This means that $L_{n}$ and $-\bar{L}_{n}$ are covariantized in the same way. This solves the aforementioned conjugation problem. Actually, the operator

$$
\Lambda_{n}^{(F)}=\mathcal{L}_{n}^{(F)}-\mathcal{L}_{n}^{(F) \dagger}=\mathcal{L}_{n}^{(F)}+\overline{\mathcal{L}}_{n}^{\left(F^{-1}\right)},
$$

is the sum of $L_{n}$ and $\bar{L}_{n}$ covariantized by means of the same conjugation

$$
\Lambda_{n}^{(F)}=F\left(L_{n}+\bar{L}_{n}\right) F^{-1},
$$

so that

$$
\exp \left(\Lambda_{n}^{(F)}\right)=F \exp \left(L_{n}+\bar{L}_{n}\right) F^{-1} .
$$

Since $\Lambda_{n}^{(F) \dagger}=-\Lambda_{n}^{(F)}$, we formally have

$$
\exp \left(\Lambda_{n}^{(F)}\right) \exp \left(\Lambda_{n}^{(F) \dagger}\right)=I=\exp \left(\Lambda_{n}^{(F) \dagger}\right) \exp \left(\Lambda_{n}^{(F)}\right) .
$$

A rigorous proof of unitarity goes as follows. Set

$$
g_{n}=\frac{\left|e_{n}(w)\right|}{\left|e_{n}(z)\right|},
$$

where $^{7} w=e^{\ell_{n}} z e^{-\ell_{n}}=\left(a_{n} z+b_{n}\right) /\left(c_{n} z+d_{n}\right)$, and note that

$$
\begin{aligned}
\exp \left(\Lambda_{n}^{(F)}\right) & =F(z, \bar{z})\left|e_{n}(z)\right|^{-1} \exp \left(\ell_{n}+\bar{\ell}_{n}\right)\left|e_{n}(z)\right| F^{-1}(z, \bar{z}) \\
& =F(z, \bar{z}) g_{n} F^{-1}(w, \bar{w}) \exp \left(\ell_{n}+\bar{\ell}_{n}\right) .
\end{aligned}
$$

\footnotetext{
${ }^{7}$ The coefficients $a_{n}, b_{n}, c_{n}$ and $d_{n}$ are given by (2.12) with $\lambda_{n}=1$ and $\lambda_{m \neq n}=0$.
} 
By

$$
\left[\ell_{n}, \exp \left(\ell_{n}\right)\right] z=0
$$

it follows that

$$
\partial_{z}\left(\exp \left(\ell_{n}\right) z\right) \exp \left(-\ell_{n}\right)=\frac{1}{z^{n+1}} \exp \left(\ell_{n}\right) z^{n+1} \exp \left(-\ell_{n}\right)
$$

that is $\partial_{z} w=e_{n}(w) / e_{n}(z)$, so that

$$
g_{n} /\left|\partial_{z} w\right|^{2}=1 / g_{n} .
$$

We then see that the operators $e^{\Lambda_{n}^{(F)}}$ are unitary

$$
\begin{aligned}
\left\langle\phi \mid e^{\Lambda_{n}^{(F)}} \psi\right\rangle & =\int_{\mathbb{H}} \frac{d \nu(w)}{\left|\partial_{z} w\right|^{2}} \bar{\phi}(z, \bar{z}) F(z, \bar{z}) g_{n} F^{-1}(w, \bar{w}) \psi(w, \bar{w}) \\
& =\int_{\mathbb{H}} d \nu(w) \overline{F(w, \bar{w}) g_{n}^{-1} F^{-1}(z, \bar{z}) \phi(z, \bar{z})} \psi(w, \bar{w}) \\
& =\left\langle e^{-\Lambda_{n}^{(F)}} \phi \mid \psi\right\rangle,
\end{aligned}
$$

where in the first equality we used the fact that $\mathrm{PSL}_{2}(\mathbb{R})$ is the automorphism group of $\mathbb{H}$.

\subsection{Selecting the Fuchsian generators}

We now digress on the possible realizations of the fundamental relation (2.5) for a Fuchsian group in the hyperbolic case. Being differential operators, the $V_{k}$ in (2.17), have the property of acting in the reverse order with respect to the matrix product. This aspect raises a subtlety in considering the relationship between $\pi_{1}(\Sigma)$ and the uniformizing group $\Gamma$. Namely, let us consider the normal form for $\Sigma$. This is a polygon whose symbol is

$$
a_{1} b_{1} a_{1}^{-1} b_{1}^{-1} \ldots a_{g} b_{g} a_{g}^{-1} b_{g}^{-1}=I
$$

where $\left\{a_{k}, b_{k}\right\}$ is a basis for $\pi_{1}(\Sigma)$. Cutting the surface along these cycles one obtains a simply connected domain whose vertices are connected by elements of the covering group. If one considers this domain as sitting on the upper half-plane, then one can consider it as a fundamental domain with the transformations connecting the vertices given by elements of $\Gamma$. Let us order the vertices of the polygon in the counterclockwise direction and denote them by $z=z_{0}, z_{1}, \ldots z_{4 g-1}, z_{4 g}=z$. We denote this fundamental domain for $\Gamma$ by

$$
\mathcal{F}_{z}[\Gamma]=\left\{z=z_{0}, z_{1}, z_{2}, \ldots, z_{4 g-1}, z_{4 g}=z\right\}
$$

with the vertices joined by geodesics. Note that since the geodesics are univocally determined it follows that the $4 g$-gon fundamental domain itself is univocally determined by the action of the Fuchsian generators on the base-point $z$.

A consequence of (5.13) is that the elements of $\Gamma$ satisfy a similar fundamental relation. Among these, the one we wrote for the $\gamma_{k}$ in (2.5), is the canonical one, that is the one in 
which the generators appear in the sequence $\gamma_{2 j-1} \gamma_{2 j} \gamma_{2 j-1}^{-1} \gamma_{2 j}^{-1}$. This version is obtained using the identification

$$
z_{1}=\gamma_{1} z, \quad z_{2}=\gamma_{1} \gamma_{2} z, \quad z_{3}=\gamma_{1} \gamma_{2} \gamma_{1}^{-1} z, \quad \ldots, \quad z_{4 g}=\prod_{j=1}^{g}\left(\gamma_{2 j-1} \gamma_{2 j} \gamma_{2 j-1}^{-1} \gamma_{2 j}^{-1}\right) z=z .
$$

Other representations can be found e.g. by Dehn twisting. ${ }^{8}$ Note that these transformations leave $\Gamma$ invariant and should not be confused with the ones obtained by conjugating $\Gamma$ in ${ }^{9} \mathrm{PSL}_{2}(\mathbb{R})$. Anyway, the usual representation for the fundamental relation satisfied by the generators of $\Gamma$ given in (2.5) does not fit with the aim of our construction. Actually, what we essentially need is to provide a central extension of the Fuchsian group. In particular, we are looking for operators provinding a projective representation of $\Gamma$, such that the fundamental relation is modified by a phase. To discuss this aspect we first need to introduce the Fuchsian matrices $\beta_{k}$ defined by

$$
z_{k}=\beta_{k} z_{k-1} \equiv \frac{\beta_{k_{11}} z_{k-1}+\beta_{k_{12}}}{\beta_{k_{21}} z_{k-1}+\beta_{k_{22}}} .
$$

Since $z_{4 g}=z$, we have that the $\beta_{k}$ satisfy the fundamental relation

$$
\beta_{4 g} \beta_{4 g-1} \ldots \beta_{1}=I \text {. }
$$

The associated operators providing a differential representation of $\Gamma$ are

$$
T_{k} \equiv T\left(\beta_{k}\right)=\exp \left(\lambda_{-1}^{(k)}\left(L_{-1}+\bar{L}_{-1}\right)\right) \exp \left(\lambda_{0}^{(k)}\left(L_{0}+\bar{L}_{0}\right)\right) \exp \left(\lambda_{1}^{(k)}\left(L_{1}+\bar{L}_{1}\right)\right)
$$

where $\lambda_{-1}^{(k)}, \lambda_{0}^{(k)}$ and $\lambda_{1}^{(k)}$ are defined in such a way that

$$
T_{k} f(z, \bar{z}) T_{k}^{-1}=f\left(\beta_{k}^{-1} z, \beta_{k}^{-1} \bar{z}\right) .
$$

The characterizing property of the generators $\beta_{k}$ for $\Gamma$, is that (5.15) allows us to associate $T_{k}$ to the geodesic connecting $z_{k-1}$ and $z_{k}$. This is an essential point because it will allow to obtain the phase of the central extension of $\Gamma$ in terms of an integral whose contour coincides with the fundamental domain. In particular, the covariantization of the $T_{k}$ will be performed by multiplying the $T_{k}$ on the left by the abelian Wilson line associated to the geodesic connecting $z_{k-1}$ and $z_{k}$. For this reason we will call these covariantized versions of the $T_{k}$ abelian Wilson-Fuchs operators. Let us note that these Wilson lines on the upper half-plane correspond to Wilson loops on the Riemann surface.

In order to derive the relationships between the $\gamma_{k}$ and $\beta_{k}$ we compare

$$
z_{1}=\gamma_{1} z, \quad z_{2}=\gamma_{1} \gamma_{2} z, \quad z_{3}=\gamma_{1} \gamma_{2} \gamma_{1}^{-1} z, \quad z_{4}=\gamma_{1} \gamma_{2} \gamma_{1}^{-1} \gamma_{2}^{-1} z, \ldots,
$$

\footnotetext{
${ }^{8}$ These correspond to in general non simultaneous conjugation of $\Gamma$ 's generators by suitable strings of the generators themselves (see for example $[13,14]$ ).

${ }^{9}$ While the conjugation in $\mathrm{PSL}_{2}(\mathbb{R})$ can be used to fix three real parameters, so that the number of real independent moduli reduces to $3 \times \sharp$ generators -3 (due to the fundamental relation) -3 (due to conjugation $)=6 g-6$, the Dehn twists correspond to a discrete set of transformations whose existence implies the nontrivial orbifold structure of the moduli space of Riemann surfaces.
} 
with

$$
z_{1}=\beta_{1} z, \quad z_{2}=\beta_{2} \beta_{1} z, \quad z_{3}=\beta_{3} \beta_{2} \beta_{1} z, \quad z_{4}=\beta_{4} \beta_{3} \beta_{2} \beta_{1} z, \ldots,
$$

to obtain

$$
\beta_{1}=\gamma_{1}, \quad \beta_{2}=\gamma_{1} \gamma_{2} \gamma_{1}^{-1}, \quad \beta_{3}=\gamma_{1} \gamma_{2} \gamma_{1}^{-1} \gamma_{2}^{-1} \gamma_{1}^{-1}, \quad \beta_{4}=\gamma_{1} \gamma_{2} \gamma_{1}^{-1} \gamma_{2}^{-1} \gamma_{1} \gamma_{2}^{-1} \gamma_{1}^{-1}, \ldots
$$

One can check that the above relationships extend to

$$
\beta_{k}=\rho_{k} \gamma_{\sigma_{k}}^{\epsilon_{k}} \rho_{k}^{-1}
$$

$k \in[1,4 g]$, where

$$
\rho_{k}=\prod_{j=1}^{k-1} \gamma_{\sigma_{j}}^{\epsilon_{j}}
$$

$\rho_{1} \equiv I$, and

$$
\epsilon_{k}=(-1)^{[(k-1) / 2]}, \quad \sigma_{k}=2\left[\frac{2 k+3-2[k / 2]}{4}\right]+2[k / 2]-k,
$$

with [.] denoting the integer part. Note that

$$
\epsilon_{4 k}=\epsilon_{4 k-1}=-1, \quad \epsilon_{4 k-2}=\epsilon_{4 k-3}=1,
$$

and

$$
\sigma_{4 k}=\sigma_{4 k-2}=2 k, \quad \sigma_{4 k-1}=\sigma_{4 k-3}=2 k-1,
$$

$k \in[1, g]$. Eq. (5.19) implies

$$
\beta_{4 k-j}=\beta_{4 k-j-1} \beta_{4 k-j-2}^{-1} \beta_{4 k-j-1}^{-1},
$$

where $j=0,1$, and $k \in[1, g]$. We can use these relationships between the $\beta_{k}$ to select $2 g$ elements, say $\beta_{4 k-3}, \beta_{4 k-2}, k \in[1, g]$ which can be seen as a complete set of generators for $\Gamma$. In particular, by (5.20) we can express $\beta_{4 k-1}, \beta_{4 k}, k \in[1, g]$ in the form

$$
\beta_{4 k-1}=\beta_{4 k-2} \beta_{4 k-3}^{-1} \beta_{4 k-2}^{-1},
$$

and

$$
\beta_{4 k}=\beta_{4 k-2} \beta_{4 k-3}^{-1} \beta_{4 k-2}^{-1} \beta_{4 k-3}^{-1} \beta_{4 k-2} .
$$

\subsection{The Wilson-Fuchs operators}

In (2.17) we introduced the operators $V_{k}$ which are defined in terms of the $\ell_{n}$. However, in order to construct unitary operators, we should use the $L_{n}$ 's rather than the $\ell_{n}$ 's. Nevertheless, since the algebras of the $\ell_{n}$ 's and $L_{n}$ 's coincide, we have that the commutation properties between the $V_{k}$, and therefore fundamental relation (2.16), would remain invariant if the $\ell_{n}$ in $V_{k}$ are replaced by the $L_{n}$ 's. Similarly the $T_{k} \equiv T\left(\beta_{k}\right)$ would satisfy the same fundamental relation under the replacement $L_{n} \rightarrow \ell_{n}, \bar{L}_{n} \rightarrow \bar{\ell}_{n}$. 
Let us now consider the operators $T_{k}$. Since their action is in the reverse order with respect to the one of the matrix product, by (5.16) and (5.18) we have

$$
T_{4 g} \ldots T_{1} f(z, \bar{z}) T_{1}^{-1} \ldots T_{4 g}^{-1}=f\left(\beta_{1}^{-1} \ldots \beta_{4 g}^{-1} z, \beta_{1} \ldots \beta_{4 g} \bar{z}\right)=f(z, \bar{z}),
$$

that is ${ }^{10}$

$$
T_{4 g} T_{4 g-1} \ldots T_{1}=I .
$$

Let $A$ be a $\mathrm{U}(1)$ connection. We set

$$
d_{A}(z, w)=\int_{z}^{w} A
$$

where the contour of integration is the Poincaré geodesic connecting $z$ and $w$. Recall that Poincaré geodesics are semi-circles centered on the real axis. Semi-circles through $\infty$ correspond to straight lines parallel to the imaginary axis. In the following we will mainly be interested in the case in which $w$ is dependent on $z$, in particular we will consider the function

$$
d_{A}(z, \mu z)=\int_{z}^{\mu z} A
$$

where

$$
\mu z=\frac{a z+b}{c z+d},
$$

$\mu \in \Gamma$. Let $b$ be an arbitrary real number. Consider the Wilson loop

$$
W_{\beta_{k}}=\exp \left(i b \int_{z}^{\beta_{k}^{-1} z} A\right)
$$

and define the Wilson-Fuchs operators

$$
\mathcal{U}_{k}=W_{\beta_{k}} T_{k},
$$

where the $T_{k}$ have been defined in (5.17). Each $\mathcal{U}_{k}$ defines the function $F_{k}(z, \bar{z})$ solution of the equation

$$
F_{k} T_{k} F_{k}^{-1}=\mathcal{U}_{k}
$$

Since by (5.18) we have

$$
T_{k} F_{k}^{-1}(z, \bar{z})=F_{k}^{-1}\left(\beta_{k}^{-1} z, \beta_{k}^{-1} \bar{z}\right) T_{k},
$$

it follows that eq. (5.25) is equivalent to

$$
F_{k}\left(\beta_{k}^{-1} z, \beta_{k}^{-1} \bar{z}\right)=\exp \left(-i b d_{A}\left(z, \beta_{k}^{-1} z\right)\right) F_{k}(z, \bar{z}) .
$$

We also note that since

$$
F_{k} \exp \left(L_{n}+\bar{L}_{n}\right) F_{k}^{-1}=\exp \left(F_{k}\left(L_{n}+\bar{L}_{n}\right) F_{k}^{-1}\right),
$$

\footnotetext{
${ }^{10}$ We observe that a possible phase on the right hand side of (5.21) is excluded by construction.
} 
it follows by (5.25) that

$$
\mathcal{U}_{k}=F_{k} T_{k} F_{k}^{-1}=\exp \left(\lambda_{-1}^{(k)} \Lambda_{-1, k}\right) \exp \left(\lambda_{0}^{(k)} \Lambda_{0, k}\right) \exp \left(\lambda_{1}^{(k)} \Lambda_{1, k}\right),
$$

where

$$
\Lambda_{n, k} \equiv \Lambda_{n, k}^{\left(F_{k}\right)}=F_{k}\left(L_{n}+\bar{L}_{n}\right) F_{k}^{-1}
$$

In particular,

$$
\exp \left(\Lambda_{n, k}\right)=F_{k} \exp \left(L_{n}+\bar{L}_{n}\right) F_{k}^{-1} .
$$

Eqs. (5.11) and (5.27) show that the $\mathcal{U}_{k}$ 's are unitary operators

$$
\mathcal{U}_{k} \mathcal{U}_{k}^{\dagger}=I=\mathcal{U}_{k}^{\dagger} \mathcal{U}_{k}
$$

Note that by (5.18) we have

$$
\mathcal{U}_{k}^{\dagger}=\mathcal{U}_{k}^{-1}=T_{k}^{-1} \exp \left(-i b \int_{z}^{\beta_{k}^{-1} z} A\right)=\exp \left(-i b \int_{\beta_{k} z}^{z} A\right) T_{k}^{-1} .
$$

\subsection{Covariantizing the generators of the uniformizing group}

We now have all the ingredients to define the covariantization of the generators of the uniformizing group. We have seen how to covariantize a given operator of $\operatorname{sl}_{2}(\mathbb{R})$. The problem now is the following. Let us write $T_{k}$ in the form

$$
T_{k}=\exp \left(D_{k}\right)
$$

Knowing $D_{k}$ allows one to define the covariant operator in the natural way, that is

$$
\mathcal{D}_{k}:=F_{k} D_{k} F_{k}^{-1}
$$

so that, by (5.27),

$$
\mathcal{U}_{k}=\exp \left(\mathcal{D}_{k}\right)
$$

On the other hand, by (5.17) we have

$$
\exp \left(D_{k}\right)=\exp \left(\lambda_{-1}^{(k)}\left(L_{-1}+\bar{L}_{-1}\right)\right) \exp \left(\lambda_{0}^{(k)}\left(L_{0}+\bar{L}_{0}\right)\right) \exp \left(\lambda_{1}^{(k)}\left(L_{1}+\bar{L}_{1}\right)\right) .
$$

It follows that in order to find $D_{k}$, and therefore $\mathcal{D}_{k}$, one needs to solve the BCH problem (5.31). On the other hand, as reviewed in section 3, the problem (5.31) belongs to the class of the new closed forms for the BCH formula derived in [4]. In particular, by $(3.18),(5.27),(5.30)$, and the fact that, for any, the $k \Lambda_{j, k}$ 's satisfy the $\mathrm{sl}_{2}(\mathbb{R})$ commutation relations, with the same normalization of the ones satisfied by the $\ell_{n}$ 's (and $L_{n}$ 's), it follows that

$$
\mathcal{D}_{k}=\frac{\lambda_{+}^{(k)}-\lambda_{-}^{(k)}}{e^{-\lambda_{-}^{(k)}}-e^{-\lambda_{+}^{(k)}}}\left[\lambda_{-1}^{(k)} \Lambda_{-1, k}+\left(2-e^{-\lambda_{+}^{(k)}}-e^{-\lambda_{-}^{(k)}}\right) \Lambda_{0, k}+\lambda_{1}^{(k)} \Lambda_{1, k}\right],
$$

where

$$
e^{-\lambda_{ \pm}^{(k)}}=\frac{1+e^{-\lambda_{0}^{(k)}}-\lambda_{-1}^{(k)} \lambda_{1}^{(k)} \pm \sqrt{\left(1+e^{-\lambda_{0}^{(k)}}-\lambda_{-1}^{(k)} \lambda_{1}^{(k)}\right)^{2}-4 e^{-\lambda_{0}^{(k)}}}}{2} .
$$


Let us explicitly rewrite $\mathcal{D}_{k}$ in terms of differentials operators. We have

$$
\begin{aligned}
\mathcal{D}_{k}=\frac{\lambda_{+}^{(k)}-}{\lambda_{-}^{(k)}} & \\
e^{-\lambda_{-}^{(k)}}- & e^{-\lambda_{+}^{(k)}} \times \\
& \times F_{k}\left[\lambda_{-1}^{(k)}\left(\partial_{z}+\partial_{\bar{z}}\right)+\left(2-e^{-\lambda_{+}^{(k)}}-e^{-\lambda_{-}^{(k)}}\right)\left(z \partial_{z}+\bar{z} \partial_{\bar{z}}+1\right)\right. \\
& \left.+\lambda_{1}^{(k)}\left(z^{2} \partial_{z}+\bar{z}^{2} \partial_{\bar{z}}+z+\bar{z}\right)\right] F_{k}^{-1} .
\end{aligned}
$$

This is a basic result. Starting with the analogy with the case of the torus, we considered several issues, such as the problem of the simultaneous covariantization, that led to consider various differential representations of $\operatorname{sl}_{2}(\mathbb{R})$, and then the problem of unitarity. This culminated with the use of the recent results on the $\mathrm{BCH}$ formula, that, finally, implied (5.34).

\subsection{Computing the phase}

Let us consider the following string of operators

$$
\mathcal{U}_{1}^{-1} \ldots \mathcal{U}_{4 g}^{-1}=\exp \left(i b \int_{z}^{\beta_{1} z} A\right) T_{1}^{-1} \exp \left(i b \int_{z}^{\beta_{2} z} A\right) T_{2}^{-1} \ldots \exp \left(i b \int_{z}^{\beta_{4 g} z} A\right) T_{4 g}^{-1} .
$$

Moving the operators $T_{k}^{-1}$ on the right we obtain

$$
\mathcal{U}_{1}^{-1} \ldots \mathcal{U}_{4 g}^{-1}=\exp \left(i b \int_{z}^{\beta_{1} z} A+i b \int_{\beta_{1} z}^{\beta_{2} \beta_{1} z} A+\ldots+i b \int_{\beta_{4 g-1} \ldots \beta_{1} z}^{\beta_{4 g} \ldots \beta_{1} z} A\right) T_{1}^{-1} \ldots T_{4 g}^{-1}
$$

that by $(5.21)$ reads

$$
\mathcal{U}_{4 g} \ldots \mathcal{U}_{1}=\exp \left(-i b \oint_{\partial \mathcal{F}_{z}[\Gamma]} A\right)
$$

where $\mathcal{F}_{z}[\Gamma]$ is the fundamental domain (5.14).

Until now the construction concerned an arbitrary $\mathrm{U}(1)$ connection $A$. We now consider the condition on $A$ in order to provide a unitary projective representation of the central extension of $\Gamma$, the integral $\oint_{\partial \mathcal{F}_{z}[\Gamma]} A$ should be $z$-independent. Let us first apply Stokes' theorem ${ }^{11}$

$$
\oint_{\partial \mathcal{F}_{z}[\Gamma]} A=\int_{\mathcal{F}_{z}[\Gamma]} d A
$$

An arbitrary transformation of a point $z$ in $\mathbb{H}$ can be expressed as $z \rightarrow z^{\prime}=\mu z$ for some $\mu \in \mathrm{PSL}_{2}(\mathbb{R})$. The fundamental domain with base-point $\mu z$ reads

$$
\mathcal{F}_{\mu z}[\Gamma]=\left\{\mu z, \beta_{1} \mu z, \beta_{2} \beta_{1} \mu z, \beta_{3} \beta_{2} \beta_{1} \mu z, \ldots\right\} .
$$

Therefore, $z$-independence implies

$$
\int_{\mathcal{F}_{\mu z}[\Gamma]} d A=\int_{\mathcal{F}_{z}[\Gamma]} d A
$$

\footnotetext{
${ }^{11}$ Recall that our choice of generators of the Fuchsian group corresponds to the boundary $\partial \mathcal{F}_{z}$ being counterclockwise oriented.
} 
We now consider the fundamental domain obtained from $\mathcal{F}_{z}[\Gamma]$ under the $\mu$ map. Since by definition the sides of $\mathcal{F}_{z}[\Gamma]$ are geodesics and these transform to geodesics under the action of $\mathrm{PSL}_{2}(\mathbb{R})$, it follows that the image of a Möbius transformation acting on the entire domain $\mathcal{F}_{z}[\Gamma]$, is uniquely fixed by the Möbius transformed vertices, that is

$$
\mu \mathcal{F}_{z}[\Gamma]=\left\{\mu z, \mu \beta_{1} z, \mu \beta_{2} \beta_{1} z, \mu \beta_{3} \beta_{2} \beta_{1} z, \ldots\right\} .
$$

The domains $\mathcal{F}_{\mu z}[\Gamma]$ and $\mu \mathcal{F}_{z}[\Gamma]$ coincide up to a conjugation of $\Gamma$ by $\mu$, namely

$$
\mathcal{F}_{\mu z}[\Gamma]=\mu \mathcal{F}_{z}\left[\mu^{-1} \Gamma \mu\right] .
$$

Since the representation cannot depend on the concrete choice of the fundamental domain, we should check that the connection $A$ satisfies

$$
\int_{\mu \mathcal{F}_{z}\left[\mu^{-1} \Gamma \mu\right]} d A=\int_{\mu \mathcal{F}_{z}[\Gamma]} d A
$$

Thus, from eqs. (5.37)(5.38) and (5.39) we have

$$
\int_{\mathcal{F}_{z}[\Gamma]} d A=\int_{\mu \mathcal{F}_{z}[\Gamma]} d A=\int_{\mathcal{F}_{z}[\Gamma]} \mu^{\star} d A
$$

that is

$$
\int_{\mathcal{F}_{z}[\Gamma]}\left(d A-\mu^{\star} d A\right)=0
$$

This imply that $d A$ must be $\mathrm{PSL}_{2}(\mathbb{R})$-invariant. It is well known that, up to an overall constant factor, the Poincaré form

$$
\omega_{P}=2 g_{z \bar{z}} d \nu=e^{\varphi} \frac{i}{2} d z \wedge d \bar{z}, \quad e^{\varphi}=y^{-2},
$$

is the unique $\mathrm{PSL}_{2}(\mathbb{R})$-invariant $(1,1)$-form. We choose the proportionality factor to be 1 , that is

$$
F=d A=2 i\left(\partial_{\bar{z}} A_{z}-\partial_{z} A_{\bar{z}}\right) d \nu=\omega_{P}
$$

that, up to gauge transformations, has solution

$$
A_{z}=A_{\bar{z}}=\frac{1}{2 y}
$$

so that

$$
A=A_{z} d z+A_{\bar{z}} d \bar{z}=\frac{d x}{y}
$$

We will call $A$ the Poincaré connection. It is worth recalling that the Poincaré metric

$$
d s^{2}=y^{-2}|d z|^{2}=2 g_{z \bar{z}}|d z|^{2},
$$

has constant negative curvature

$$
R=-g^{z \bar{z}} \partial_{z} \partial_{\bar{z}} \ln g_{z \bar{z}}=-1,
$$


that is, $\varphi$ satisfies the Liouville equation

$$
\partial_{\bar{z}} \partial_{z} \varphi=\frac{e^{\varphi}}{2} .
$$

This implies the following properties for $A$

$$
A_{z}=A_{\bar{z}}=\frac{1}{2} e^{\varphi / 2}=-\frac{i}{2} \partial_{z} \varphi=\frac{i}{2} \partial_{\bar{z}} \varphi=\frac{1}{\sqrt{2}}\left(\partial_{z} \partial_{\bar{z}} \varphi\right)^{1 / 2},
$$

and

$$
F=d A=4 A_{z} A_{\bar{z}} d \nu .
$$

From $\sqrt{g} R=-\frac{1}{2} e^{\varphi}$ and the Gauss-Bonnet theorem we have

$$
\int_{\mathcal{F}_{z}[\Gamma]} \omega_{P}=-2 \pi \chi(\Sigma)
$$

where $\chi(\Sigma)=2-2 g$ is the Euler characteristic of $\Sigma$. By (5.36), (5.40) and (5.42), we have

$$
\oint_{\partial \mathcal{F}_{z}[\Gamma]} A=-2 \pi \chi(\Sigma)
$$

and (5.35) finally becomes

$$
\mathcal{U}_{4 g} \ldots \mathcal{U}_{1}=\exp (2 \pi i b \chi(\Sigma))
$$

\subsection{Nonabelian extension}

Up to now we considered the case in which the connection is Abelian. However, it is easy to extend our construction to the nonabelian case in which the gauge group $\mathrm{U}(1)$ is replaced by $\mathrm{U}(N)$. The operators $\mathcal{U}_{k}$ now become the path-ordered exponentials

$$
\mathcal{U}_{k}=P \exp \left(i b \int_{z}^{\beta_{k}^{-1} z} A\right) T_{k}
$$

where the $T_{k}$ are the same as before, times the $\mathrm{U}(N)$ identity matrix. Eq. (5.35) is replaced by

$$
\mathcal{U}_{4 g} \ldots \mathcal{U}_{1}=P \exp \left(-i b \oint_{\partial \mathcal{F}_{z}[\Gamma]} A\right) .
$$

Given an integral along a closed contour $\sigma_{z}$ with base-point $z$, the path-ordered exponentials for a connection $A$ and its gauge transform $A^{U}=U^{-1} A U+U^{-1} d U$ are related by [15]

$$
\begin{aligned}
P \exp \left(i \oint_{\sigma_{z}} A\right) & =U(z) P \exp \left(i \oint_{\sigma_{z}} A^{U}\right) U^{-1}(z) \\
& =U(z) P\left[\exp \left(i \oint_{\sigma_{z}} d \sigma^{\mu} \int_{0}^{1} d s s \sigma^{\nu} U^{-1}(s \sigma) F_{\nu \mu}(s \sigma) U(s \sigma)\right)\right] U^{-1}(z) .
\end{aligned}
$$

This implies that the only possibility to get a coordinate-independent phase is for the curvature $(1,1)$-form $F=d A+[A, A] / 2$ to be the identity matrix in the gauge indices times a $(1,1)$-form $\eta$, that is

$$
F=\eta I
$$


It follows that

$$
P \exp \left(-i b \oint_{\partial \mathcal{F}_{z}[\Gamma]} A\right)=\exp \left(-i b \int_{\mathcal{F}_{z}[\Gamma]} F\right)
$$

This is only a necessary condition for coordinate-independence. Reasoning as in the Abelian case, one concludes that $\eta$ should be proportional to the Poincaré $(1,1)$-form, that is

$$
\eta=k \omega_{P} .
$$

In order to fix the constant $k$, we first consider the vector bundle $\mathcal{E}$ on which the connection $A$ is defined. Taking the gauge group $\mathrm{U}(N)$ in the fundamental representation, the dimension $N$ of the vector fiber is called the rank of $\mathcal{E}$. The degree is the integral of the first Chern class $^{12}$

$$
M=\operatorname{deg}(\mathcal{E})=\frac{1}{2 \pi} \operatorname{tr} \int_{\mathcal{F}} F,
$$

where, to simplify notation we used $\mathcal{F}$ instead of $\mathcal{F}_{z}[\Gamma]$. Since in our case the trace gives a factor $N$, we have

$$
\int_{\mathcal{F}} F=2 \pi \mu(\mathcal{E}) I
$$

where

$$
\mu(\mathcal{E})=\frac{\operatorname{deg}(\mathcal{E})}{\operatorname{rank}(\mathcal{E})}=\frac{M}{N} .
$$

Thus, by (5.44) and (5.46) we have

$$
\mathcal{U}_{4 g} \ldots \mathcal{U}_{1}=\exp (-2 \pi i b \mu(\mathcal{E})) I .
$$

Finally, we observe that by (5.42) and (5.48) it follows that the constant in (5.47) is $k=-\mu(\mathcal{E}) / \chi(\Sigma)$, that is

$$
F=-\frac{\mu(\mathcal{E})}{\chi(\Sigma)} \omega_{P} I
$$

\section{The gauge length}

By (5.41) it follows that eq. (5.22) becomes

$$
d_{A}(z, w)=\int_{z}^{w} \frac{d x}{y},
$$

where, we recall, the contour integral is along the Poincaré geodesic connecting $z$ and $w$. Let us denote by $x_{0}$ the center of this geodesic and $\rho$ its radius. In polar coordinates we have $z-x_{0}=\rho e^{i \alpha_{z}}, w-x_{0}=\rho e^{i \alpha_{w}}$ and $d x / y=\rho d \cos \alpha / \rho \sin \alpha=-d \alpha$, so that

$$
d_{A}(z, w)=-\int_{\alpha_{z}}^{\alpha_{w}} d \alpha=\alpha_{z}-\alpha_{w}
$$

Since

$$
e^{i \alpha_{z w}}=\frac{e^{i \alpha_{z}}-e^{-i \alpha_{w}}}{e^{i \alpha_{w}}-e^{-i \alpha_{z}}}=\frac{z-\bar{w}}{w-\bar{z}}
$$

\footnotetext{
${ }^{12}$ Our convention for $A$ differs from the one in the mathematical literature by a factor $i$.
} 
where $\alpha_{z w} \equiv \alpha_{z}-\alpha_{w}$, we have

$$
d_{A}(z, w)=\alpha_{z w}=-i \ln \left(\frac{z-\bar{w}}{w-\bar{z}}\right) .
$$

Note that in terms of $z$ and $w$, we have

$$
x_{0}=\frac{1}{2} \frac{|w|^{2}-|z|^{2}}{\Re(w-z)},
$$

which shows the dependence of $\alpha_{z}$ (and $\alpha_{w}$ ) on $z$ and $w$. Also note that both $\alpha_{z}$ and $\alpha_{w}$ range between 0 and $\pi$, with the extremes corresponding to points on the extended real axis $\mathbb{R} \cup\{\infty\}=\partial \mathbb{H}$.

\subsection{The gauge length as pseudo-distance}

We now show that

$$
\ell_{A}(z, w)=\left|d_{A}(z, w)\right|,
$$

is in fact a pseudo-distance that we call gauge length. The symmetry property follows from the antisymmetry of $d_{A}(z, w)$ while the triangle inequality

$$
\ell_{A}\left(z_{1}, z_{2}\right)+\ell_{A}\left(z_{2}, z_{3}\right) \geq \ell_{A}\left(z_{1}, z_{3}\right),
$$

follows from the fact that $\ell_{A}(z, w)=\left|\alpha_{z w}\right|$ or, equivalently, from the observation that $\ell_{A}\left(z_{1}, z_{2}\right)+\ell_{A}\left(z_{2}, z_{3}\right)-\ell_{A}\left(z_{1}, z_{3}\right)$ is the Poincaré area of the geodesic triangle with vertices $z_{1}, z_{2}$ and $z_{3}$. Since

$$
\ell_{A}(z, w)=0 \quad \text { iff } \quad \Re(z)=\Re(w),
$$

it follows that $\ell_{A}(z, w)$ cannot be a distance.

Note that eq. (6.4), seen as an inequality involving angles, is similar to the one satisfied by the angles of triangles in Euclidean geometry. While in Euclidean geometry the Schwarz inequality is satisfied both by the angles and by the lengths of the sides of triangles, in the case of hyperbolic geometry, the gauge length coincides with the angles themselves.

Another property of this pseudo-distance is that, as we said, it has $\pi$ as upper bound corresponding to the case in which the two points are on the real axis, so that

$$
\ell_{A}(z, w)<\pi, \quad \forall(z, w) \in \mathbb{H}^{2} .
$$

\subsection{The gauge length as Poincaré area}

The gauge length has some interesting properties which are worth mentioning. For example, while the geodesic distance between a point in $\mathbb{H}$ and one on the real axis measured with respect to the Poincaré metric is divergent, the corresponding gauge distance is finite. As a consequence, measuring the gauge distance between one point on a Riemann surface and a puncture on it gives a finite result. In particular, the greatest gauge length between two points is $\pi$, which is the gauge distance between two punctures. Also, the lower bound for $d_{A}(z, w)$ is 0 , which corresponds to the case in which two points have the same real part. 
We saw that since $d A=e^{\varphi} d \nu$ is the infinitesimal Poincaré area, Stokes' theorem and Gauss-Bonnet formula give $\oint_{\partial \mathcal{F}} A=-2 \pi \chi(\Sigma)$. The Stokes formula is also useful to describe the gauge length of a single geodesic as Poincare area. In this respect recall that the Poincaré area of a hyperbolic triangle of angles $\alpha, \beta$ and $\gamma$, is $\pi-\alpha-\beta-\gamma$. Then consider the geodesic triangle $\mathcal{D}$ corresponding to the geodesic joining $z$ and $w$, together with the two geodesics connecting $z$ and $w$ to the point at imaginary infinity, which is a cusp so that $\gamma=0$. The latter two geodesics correspond to straight lines parallel to the imaginary axis, thus they have vanishing gauge length. Then, by Stokes' theorem

$$
\ell_{A}(z, w)=\left|\int_{z}^{w} A\right|=\int_{\partial \mathcal{D}} A=\int_{\mathcal{D}} d A=\pi-\alpha-\beta
$$

giving the relation

$$
\alpha_{z w}=\pi-\alpha-\beta,
$$

that can be directly verified. Therefore, the gauge length has in fact properties which are related to those of an area function.

An interesting property of the gauge length concerns its transformation properties under $\mathrm{PSL}_{2}(\mathbb{R})$ Möbius transformations. We have

$$
d_{A}(\mu z, \mu w)=d_{A}(z, w)+\frac{i}{2} \ln \frac{\bar{\mu}_{z} \mu_{w}}{\mu_{z} \bar{\mu}_{w}}
$$

where

$$
\mu x \equiv \frac{a x+b}{c x+d},
$$

and

On the other hand

$$
\mu_{x} \equiv \partial_{x} \mu x=\frac{1}{(c x+d)^{2}} .
$$

$$
\frac{i}{2} \ln \frac{\bar{\mu}_{z} \mu_{w}}{\mu_{z} \bar{\mu}_{w}}=d_{A}(0, c z+d)+d_{A}(c w+d, 0)
$$

showing that the Möbius transformation of the integration limits corresponds to adding the gauge lengths between 0 and $c z+d$ and between $c w+d$ and 0

$$
\int_{\mu z}^{\mu w} A=\int_{z}^{w} A+\int_{0}^{\mu_{z}^{-1 / 2}} A+\int_{\mu_{w}^{-1 / 2}}^{0} A .
$$

In this respect it is worth noticing that if $c>0$ then the points $\mu_{z}^{-1 / 2}=(c z+d) \in \mathbb{H}$ and $\mu_{w}^{-1 / 2}=(c w+d) \in \mathbb{H}$ are hyperbolic transformations of $z$ and $w$ respectively, that is $\mu_{z}^{-1 / 2}=\nu z, \mu_{w}^{-1 / 2}=\nu w$ where

$$
\nu=\left(\begin{array}{cc}
c^{1 / 2} & b c^{1 / 2} \\
0 & c^{-1 / 2}
\end{array}\right) .
$$

Denote by $z_{k}, k=1, \ldots, n, n \geq 3$, the vertices of an $n$-gon with angles $\alpha_{1}, \ldots, \alpha_{n}$. Its gauge length is given by

$$
\ell_{A}^{(n)}\left(\left\{z_{k}\right\}\right)=\sum_{k=1}^{n}\left|\alpha_{z_{k} z_{k+1}}\right|=\pi(n-2)-\sum_{k=1}^{n} \alpha_{k},
$$


where $z_{n+1} \equiv z_{1}$. Eq. (6.5) implies that the gauge length of an $n$-gon is $\mathrm{PSL}_{2}(\mathbb{C})$-invariant, that is

$$
\ell_{A}^{(n)}\left(\left\{\mu z_{k}\right\}\right)=\ell_{A}^{(n)}\left(\left\{z_{k}\right\}\right)
$$

This invariance can be also seen by observing that $\ell_{A}^{(n)}$ can be expressed in terms of cross ratios. In particular, for the geodesic triangle we have

$$
\ell_{A}^{(3)}=\left|\ln \left(z_{1}, z_{2}, \bar{z}_{2}, \bar{z}_{3}\right)\left(z_{2}, z_{3}, \bar{z}_{2}, \bar{z}_{1}\right)\right|
$$

where

$$
\left(x_{1}, x_{2}, x_{3}, x_{4}\right)=\frac{x_{1}-x_{3}}{x_{1}-x_{4}} \frac{x_{2}-x_{4}}{x_{2}-x_{3}} .
$$

The invariance $(6.6)$ is then a consequence of the $\mathrm{PSL}_{2}(\mathbb{C})$-invariance

$$
\left(\mu x_{1}, \mu x_{2}, \mu x_{3}, \mu x_{4}\right)=\left(x_{1}, x_{2}, x_{3}, x_{4}\right)
$$

which together with the fact that any cyclic permutation corresponds to an involution

$$
\left(x_{4}, x_{1}, x_{2}, x_{3}\right)=\frac{\left(x_{1}, x_{2}, x_{3}, x_{4}\right)}{\left(x_{1}, x_{2}, x_{3}, x_{4}\right)-1}
$$

constitute the main properties of the cross ratio.

\subsection{Möbius transformations as gauge transformations}

While on one hand eq. (5.36) implies the equality

$$
\oint_{\partial \mathcal{F}} A=\int_{\mathcal{F}} \omega_{P}
$$

on the other hand the Poincaré metric is $\mathrm{PSL}_{2}(\mathbb{R})$-invariant while $A$ is not. As a consequence the variation of $A$ under a $\mathrm{PSL}_{2}(\mathbb{R})$ transformation can only be a total derivative. This is in fact the case, as

$$
A(\mu z, \mu \bar{z})=A(z, \bar{z})-i \partial_{z} \ln (c z+d) d z+i \partial_{\bar{z}} \ln (c \bar{z}+d) d \bar{z} .
$$

Since $c z+d$ has no zeroes on $\mathbb{H}$, it follows that $\ln (c z+d)$ is well defined, so that the inhomogeneous term in (6.7) can be expressed as an external derivative on $\mathbb{H}$, that is

$$
A(\mu z, \mu \bar{z})=A(z, \bar{z})+d \ln \left(\mu_{z} / \bar{\mu}_{z}\right)^{\frac{i}{2}},
$$

and

$$
\int_{z}^{w} A(\mu x, \mu \bar{x})=\int_{z}^{w} A(x, \bar{x})+\frac{i}{2} \ln \frac{\bar{\mu}_{z} \mu_{w}}{\mu_{z} \bar{\mu}_{w}}=\int_{\mu z}^{\mu w} A(x, \bar{x}) .
$$

Therefore, the isometry group of the Poincaré metric, which in turn coincides with the automorphism group of the upper half-plane, induces, when acting on the Poincaré connection $A$ itself, the gauge transformation $A \rightarrow A+d \chi$, with $\chi(z)=\ln \left(\mu_{z} / \bar{\mu}_{z}\right)^{\frac{i}{2}}$. 


\section{Acknowledgments}

It is a pleasure to thank Gaetano Bertoldi and José Isidro for collaboration in the early stage of this work. We also thank Giulio Bonelli and Dima Sorokin for interesting discussions and Roberto Volpato for enlightening discussions and careful reading of the paper.

Open Access. This article is distributed under the terms of the Creative Commons Attribution License (CC-BY 4.0), which permits any use, distribution and reproduction in any medium, provided the original author(s) and source are credited.

\section{References}

[1] Y. Frishman and J. Sonnenschein, Non-perturbative field theory: from two-dimensional conformal field theory to QCD in four dimensions, Cambridge University Press, Cambridge U.K. (2010).

[2] G. Bertoldi, J.M. Isidro, M. Matone and P. Pasti, Noncommutative Riemann surfaces, PoS (TMR99) 022 [hep-th/0003131] [INSPIRE].

[3] G. Bertoldi, J.M. Isidro, M. Matone and P. Pasti, The concept of a noncommutative Riemann surface, Phys. Lett. B 484 (2000) 323 [hep-th/0003200] [INSPIRE].

[4] M. Matone, An algorithm for the Baker-Campbell-Hausdorff formula, JHEP 05 (2015) 113 [arXiv: 1502.06589] [INSPIRE].

[5] M. Matone, Classification of commutator algebras leading to the new type of closed Baker-Campbell-Hausdorff formulas, arXiv:1503.08198 [INSPIRE].

[6] M. Matone, Closed form of the Baker-Campbell-Hausdorff formula for semisimple complex Lie algebras, arXiv:1504.05174 [INSPIRE].

[7] A. Van-Brunt and M. Visser, Special-case closed form of the Baker-Campbell-Hausdorff formula, J. Phys. A 48 (2015) 225207 [arXiv:1501.02506] [INSPIRE].

[8] A. Van-Brunt and M. Visser, Simplifying the Reinsch algorithm for the Baker-Campbell-Hausdorff series, arXiv:1501.05034 [INSPIRE].

[9] L. Bonora, M. Matone, F. Toppan and K. Wu, b-c system approach to minimal models. 1. The genus zero case, Phys. Lett. B 224 (1989) 115 [INSPIRE].

[10] L. Bonora, M. Matone, F. Toppan and K. Wu, Real weight b-c systems and conformal field theory in higher genus, Nucl. Phys. B 334 (1990) 717 [InSPIRE].

[11] A.A. Voronov, A unified approach to string scattering amplitudes, Commun. Math. Phys. 131 (1990) 179 [INSPIRE].

[12] C.G. Callan Jr. and F. Wilczek, Infrared behavior at negative curvature, Nucl. Phys. B 340 (1990) 366 [INSPIRE].

[13] H.M. Farkas and I. Kra, Riemann surfaces, $2^{\text {nd }}$ edition, Springer, Germany (1992).

[14] S.P. Novikov, Topology, Springer, Germany (1996).

[15] G. Thompson, 1992 Trieste lectures on topological gauge theory and Yang-Mills theory, hep-th/9305120 [INSPIRE]. 